\title{
QUASI-INVARIANT GAUSSIAN MEASURES FOR THE TWO-DIMENSIONAL DEFOCUSING CUBIC NONLINEAR WAVE EQUATION
}

\author{
TADAHIRO OH AND NIKOLAY TZVETKOV
}

\begin{abstract}
We study the transport properties of the Gaussian measures on Sobolev spaces under the dynamics of the two-dimensional defocusing cubic nonlinear wave equation (NLW). Under some regularity condition, we prove quasi-invariance of the mean-zero Gaussian measures on Sobolev spaces for the NLW dynamics. We achieve this goal by introducing a simultaneous renormalization on the energy functional and its time derivative and establishing a renormalized energy estimate in the probabilistic setting.
\end{abstract}

\section{INTRODUCTION}

1.1. General context. In probability theory, the transport properties of Gaussian measures under linear and nonlinear transformations have attracted wide attention since the seminal work of Cameron-Martin [3]. In the special case of linear transformations given by the translation by a fixed (deterministic) vector, Cameron-Martin provided a complete answer to this question in [3]. This result then formed the basis of the infinite dimensional analysis, the so-called Malliavin calculus. In [22, Ramer further studied the transport property of Gaussian measures under a general nonlinear transformation on an abstract Wiener space and gave a criterion, guaranteeing that Gaussian measures are quasi-invariant under general transformations which are (essentially speaking) Hilbert-Schmidt perturbations of the identity. Here, by quasi-invariance, we mean that a measure $\mu$ on a measure space $(X, \mu)$ and the pushforward $T_{*} \mu$ of $\mu$ under a measurable transformation $T: X \rightarrow X$, defined by $T_{*} \mu=\mu \circ T^{-1}$, are equivalent, namely mutually absolutely continuous with respect to each other.

The quasi-invariance result by Ramer is of course more general than Cameron-Martin's result because it applies to general nonlinear transformations and it is certainly the best result one can expect in the context of general nonlinear transformations. In [4, 5], Cruzeiro studied flows generated by vector fields on abstract Wiener spaces and established an abstract criterion, guaranteeing quasi-invariance of Gaussian measures under such flows. We point out that the verification of such a criterion was not carried out for concrete examples in [4, 5]. Lastly, let us mention a generalization of Cruzeiro's work by Peters [20, 21]. In particular, by exploiting the symplectic structure of the vector field, he also showed that

2010 Mathematics Subject Classification. 35L71; 60H30.

Key words and phrases. nonlinear wave equation; nonlinear Klein-Gordon equation; Gaussian measure; quasi-invariance. 
the Gaussian measure on $1 H^{\frac{1}{2}}(\mathbb{T}) \times H^{-\frac{1}{2}}(\mathbb{T})$ is quasi-invariant under the flow of the Wick ordered sine-Gordon equation on the circle.

In the recent works [27, 18, 14, we further studied the transport property of Gaussian measures under nonlinear Hamiltonian PDE dynamics and succeeded to prove quasiinvariance of Gaussian measures on periodic functions. In particular, in [27, the second author introduced a general strategy, combining PDE and stochastic analysis to prove quasi-invariance of Gaussian measures under nonlinear Hamiltonian PDE dynamics, thus verifying an assumption of the type imposed in [4, 5, 20, for some concrete examples (without relying on a special structure of an underlying space such as the symplectic structure in [21]). In [27], we considered the BBM-type equations and by exploiting energy estimates, which are quite standard in the field of hyperbolic PDEs, we established quasi-invariance of Gaussian measures on periodic functions, going beyond Ramer's result. While it was only stated in a remark, similar quasi-invariance results hold for the one-dimensional nonlinear wave equations (NLW) and nonlinear Klein-Gordon equations (NLKG). In [18, 14, we studied the quasi-invariance property of Gaussian measures under the dynamics of the one-dimensional cubic fourth order nonlinear Schrödinger equation. By applying gauge transformation 2 and (an infinite iteration of) normal form transformations, we proved quasi-invariance of Gaussian measures, which is optimal in terms of Sobolev regularities.

In the present paper, we will further develop the method of [27, 18] in the context of twodimensional nonlinear wave equations. We follow the new strategy introduced by the second author in [27. Namely, we prove the quasi-invariance property for a weighted Gaussian measure which is absolutely continuous with respect to the underlying Gaussian measure. The density of such a weighted Gaussian measure is inspired by an energy functional associated to the equation. Observe that our approach is already quite different compared to Ramer's analysis [22]. In a sharp contrast with the previous works [27, 18, 14], in this work, we need to use a renormalized energy functional. Such a renormalized energy is closely related to renormalizations considered in Euclidean quantum field theory [23]. On the one hand, such renormalizations often force us to work with renormalized equations. See [16] in the context of two-dimensional NLW endowed with Gibbs measures. On the other hand, this is not the case in our analysis; we are able to keep the original equation despite the use of the renormalized energy. This is achieved by performing a simultaneous renormalization of the energy functional and its time derivative. See Subsection 1.4 below. In particular, after introducing the renormalized energy, we establish a renormalized energy estimate that is suitable for studying the dynamical property of the original equation in the probabilistic manner. This renormalized energy estimate is the main novelty of this work. As we shall see below, its proof is quite intricate and it does not result from purely linear Gaussian considerations unlike the previous works [27, 18].

\footnotetext{
${ }^{1}$ More precisely, Peters considered the Gaussian measure $d \mu=Z^{-1} \exp \left(-\frac{1}{2}\|(u, v)\|_{H^{\frac{1}{2}} \times H^{-\frac{1}{2}}}^{2}\right) d u d v$ on $H^{\sigma}(\mathbb{T}) \times H^{\sigma-1}(\mathbb{T}), \sigma<0$, for which $H^{\frac{1}{2}}(\mathbb{T}) \times H^{-\frac{1}{2}}(\mathbb{T})$ is the Cameron-Martin space. See (1.4) below. Note that the regularity $\frac{1}{2}$ plays an important role in 21 since $H^{\frac{1}{2}}(\mathbb{T}) \times H^{-\frac{1}{2}}(\mathbb{T})$ is the symplectic space for the Klein-Gordon equations, including the sine-Gordon equation.

${ }^{2}$ In a recent paper [17, by applying a further gauge transformation, we extended the quasi-invariance result to the cubic nonlinear Schrödinger equation with third order dispersion.
} 
1.2. Main result. Consider the defocusing cubic nonlinear wave equation on $\mathbb{T}^{2}=(\mathbb{R} / \mathbb{Z})^{2}$ :

$$
\partial_{t}^{2} u-\Delta u+u^{3}=0
$$

where $u: \mathbb{T}^{2} \times \mathbb{R} \rightarrow \mathbb{R}$ is the unknown function. With $v=\partial_{t} u$, we rewrite (1.1) as the following first order system:

$$
\left\{\begin{array}{l}
\partial_{t} u=v \\
\partial_{t} v=\Delta u-u^{3}
\end{array}\right.
$$

The system (1.2) is a Hamiltonian system of PDEs with the Hamiltonian:

$$
H(u, v)=\frac{1}{2} \int_{\mathbb{T}^{2}}\left(|\nabla u|^{2}+v^{2}\right) d x+\frac{1}{4} \int_{\mathbb{T}^{2}} u^{4} d x .
$$

It is easy to verify that, if $(u, v)$ is a smooth solution to (1.2), then

$$
\frac{d}{d t} H(u(t), v(t))=0
$$

In view of the structure of the Hamiltonian $H(u, v)$ and the properties of the linear wave equation, it is natural to study (1.2) in the space:

$$
\mathcal{H}^{s}\left(\mathbb{T}^{2}\right) \equiv H^{s}\left(\mathbb{T}^{2}\right) \times H^{s-1}\left(\mathbb{T}^{2}\right),
$$

where $H^{s}\left(\mathbb{T}^{2}\right)$ is the classical $L^{2}$-based Sobolev space of order $s$. By a classical argument (see the next section), one can show that (1.2) is globally well-posed in $\mathcal{H}^{\sigma}\left(\mathbb{T}^{2}\right), \sigma \geq 1$. Let us denote this global flow by $\Phi_{\mathrm{NLW}}(t), t \in \mathbb{R}$.

Our main goal is to study the quasi-invariance property under $\Phi_{\mathrm{NLW}}(t)$ of the Gaussian measure $\mu_{s}$, formally defined by

$$
\begin{aligned}
d \mu_{s} & =Z_{s}^{-1} e^{-\frac{1}{2}\|(u, v)\|_{\mathcal{H}^{s+1}}^{2}} d u d v \\
& =Z_{s}^{-1} \prod_{n \in \mathbb{Z}^{2}} e^{-\frac{1}{2}\langle n\rangle^{2(s+1)}\left|\widehat{u}_{n}\right|^{2}} e^{-\frac{1}{2}\langle n\rangle^{2 s}\left|\widehat{v}_{n}\right|^{2}} d \widehat{u}_{n} d \widehat{v}_{n},
\end{aligned}
$$

where $\langle\cdot\rangle=\left(1+|\cdot|^{2}\right)^{\frac{1}{2}}$ and $\widehat{u}_{n}$ and $\widehat{v}_{n}$ denote the Fourier transforms of $u$ and $v$, respectively. Note that this measure is naturally associated to the linear wave dynamics. In particular, $\mu_{s}$ is invariant under the linear wave dynamics.

We can define the measure $\mu_{s}$ in a rigorous manner by viewing it as the induced probability measure under the map:

$$
\omega \in \Omega \longmapsto\left(u^{\omega}, v^{\omega}\right),
$$

where $u^{\omega}$ and $v^{\omega}$ are given by ${ }^{3}$

$$
u^{\omega}(x)=\sum_{n \in \mathbb{Z}^{2}} \frac{g_{n}(\omega)}{\langle n\rangle^{s+1}} e^{i n \cdot x} \quad \text { and } \quad v^{\omega}(x)=\sum_{n \in \mathbb{Z}^{2}} \frac{h_{n}(\omega)}{\langle n\rangle^{s}} e^{i n \cdot x} .
$$

Here, $\left\{g_{n}\right\}_{n \in \mathbb{Z}^{2}}$ and $\left\{h_{n}\right\}_{n \in \mathbb{Z}^{2}}$ are two sequences of "independent standard" complex-valued Gaussian random variables on a probability space $(\Omega, \mathcal{F}, P)$ conditioned that $g_{-n}=\overline{g_{n}}$, $h_{-n}=\overline{h_{n}}$. More precisely, with the index set $\Lambda$ defined by

$$
\Lambda=\left(\mathbb{Z} \times \mathbb{Z}_{+}\right) \cup\left(\mathbb{Z}_{+} \times\{0\}\right) \cup\{(0,0)\},
$$

\footnotetext{
${ }^{3}$ Henceforth, we drop the harmless factor $2 \pi$.
} 
we define $\left\{g_{n}, h_{n}\right\}_{n \in \Lambda}$ to be a sequence of independent standard complex-valued Gaussian random variables (with $g_{0}, h_{0}$ real-valued) and set $g_{-n}=\overline{g_{n}}, h_{-n}=\overline{h_{n}}$ for $n \in \mathbb{Z}^{2}$.

The partial sums of the series in (1.5) are a Cauchy sequence in $L^{2}\left(\Omega ; \mathcal{H}^{\sigma}\left(\mathbb{T}^{2}\right)\right)$ for every $\sigma<s$ and therefore one can view $\mu_{s}$ as a probability measure on $\mathcal{H}^{\sigma}\left(\mathbb{T}^{2}\right)$ for a fixed $\sigma<s$. In particular, for $s>1$, the flow $\Phi_{\mathrm{NLW}}(t)$ is well defined $\mu_{s}$-almost surely. We also point out that, for the same range of $\sigma$, the triplet $\left(\mathcal{H}^{s+1}\left(\mathbb{T}^{2}\right), \mathcal{H}^{\sigma}\left(\mathbb{T}^{2}\right), \mu_{s}\right)$ forms an abstract Wiener space. See [8, 11].

We now state our main result.

Theorem 1.1. Let $s \geq 2$ be an even integer. Then, $\mu_{s}$ is quasi-invariant under $\Phi_{\mathrm{NLW}}(t)$.

We next consider the defocusing cubic nonlinear Klein-Gordon equation:

$$
\partial_{t}^{2} u-\Delta u+u+u^{3}=0,
$$

where $u: \mathbb{T}^{2} \times \mathbb{R} \rightarrow \mathbb{R}$. As in the case of NLW, we rewrite (1.7) as the first order system:

$$
\left\{\begin{array}{l}
\partial_{t} u=v \\
\partial_{t} v=\Delta u-u-u^{3} .
\end{array}\right.
$$

The system (1.8) is a Hamiltonian system of PDEs with the Hamiltonian:

$$
E(u, v)=\frac{1}{2} \int_{\mathbb{T}^{2}}\left(u^{2}+|\nabla u|^{2}+v^{2}\right) d x+\frac{1}{4} \int_{\mathbb{T}^{2}} u^{4} d x
$$

and one directly verifies that, if $(u, v)$ is a smooth solution to (1.8), then

$$
\frac{d}{d t} E(u(t), v(t))=0 \text {. }
$$

We again have that (1.8) is globally well-posed in $\mathcal{H}^{\sigma}\left(\mathbb{T}^{2}\right), \sigma \geq 1$ (see Lemma 2.1 below). Let us denote this global flow by $\Phi_{\mathrm{NLKG}}(t), t \in \mathbb{R}$. Then, we have the following statement.

Theorem 1.2. Let $s \geq 2$ be an even integer. Then, $\mu_{s}$ is quasi-invariant under $\Phi_{\mathrm{NLKG}}(t)$.

While the proofs of Theorem 1.1 and Theorem 1.2 are very similar, it is more convenient to first prove Theorem 1.2. Hence, we shall discuss the proof of Theorem 1.2 in details and we will indicate the needed modifications leading to the proof of Theorem 1.1 in the last section of the paper.

1.3. Remarks \& comments. The restriction that $s$ is an even integer in Theorems 1.1 and 1.2 is not essential. We strongly believe that our proof together with some classical (in the field of dispersive PDEs) fractional Leibniz rule considerations provides quasi-invariance of $\mu_{s}$ for every $s \geq 2$. The extension of Theorems 1.1 and 1.2 to $s<2$ may also be tractable by incorporating some of the recent development in the low regularity probabilistic wellposedness of NLW and NLKG In order to highlight our renormalization argument, we decided not to pursue these extensions here. Similarly, we believe that our argument is applicable to the defocusing nonlinearities of higher degrees. For the conciseness of the presentation, however, we only work with the cubic nonlinearity. We also point out that our argument does not extend to the three-dimensional case. The proof of the main results

\footnotetext{
${ }^{4}$ For example, the work [16] on the invariant Gibbs measure for the 2- $d$ NLKG implies quasi-invariance of $\mu_{0}$ under the renormalized NLKG dynamics. For $\mu_{s}$ with $s>0$, one should not need the renormalized equation.
} 
(Theorems 1.1 and 1.2) in the two-dimensional case is based on a simultaneous renormalization of the energy functional and its time derivative (Subsection 1.4), which allows us to (i) construct a weighted Gaussian measure associated to the renormalized energy (Section (3) and (ii) establish a renormalized energy estimate (Theorem 1.6), controlling the time derivative of the renormalized energy. As we point out in Remarks 3.6 and 4.1, both (i) and (ii) fail in the three-dimensional case. It would be of great interest to investigate the three-dimensional case by possibly introducing a further (simultaneous) renormalization.

In [18, 14], we studied the cubic fourth order nonlinear Schrödinger equation on the circle:

$$
i \partial_{t} u=\partial_{x}^{4} u+|u|^{2} u
$$

and proved quasi-invariance of the Gaussian measure $\nu_{s}$ on $L^{2}(\mathbb{T})$ formally defined by

$$
d \nu_{s}=Z_{s}^{-1} e^{-\frac{1}{2}\|u\|_{H^{s}}^{2}} d u=Z_{s}^{-1} \prod_{n \in \mathbb{Z}} e^{-\frac{1}{2}\langle n\rangle^{2 s}\left|\widehat{u}_{n}\right|^{2}} d \widehat{u}_{n},
$$

provided that $s>\frac{1}{2}$. In [14, we also showed that the dispersion is essential for this quasi-invariance result. More precisely, we considered the following dispersionless model on $\mathbb{T}$ :

$$
i \partial_{t} u=|u|^{2} u
$$

and showed that the Gaussian measure $\nu_{s}$ is not quasi-invariant under the flow of (1.11). In a similar manner, we believe that the dispersive term is crucial in order to establish the quasi-invariance result in Theorem 1.1, no matter how large $s$ is. It is quite likely that the method of [14] can be adapted to show that the transport of $\mu_{s}$ under the (well defined) flow of

$$
\left\{\begin{array}{l}
\partial_{t} u=v \\
\partial_{t} v=-u^{3}
\end{array}\right.
$$

is not equivalent to $\mu_{s}$ (for non-trivial times). Indeed, we expect that the flow of (1.12) introduces fast time oscillations, modifying some fine regularity properties which hold true typically with respect to the Gaussian measure $\mu_{s}$.

As it is well known, the solutions to NLW can be decomposed as the linear evolution plus a "one-derivative smoother term". On the other hand, the typical Sobolev regularity on the support of $\mu_{s}$ is $\mathcal{H}^{\sigma}\left(\mathbb{T}^{2}\right), \sigma<s$. The Cameron-Martin theorem in this context states that for a fixed $\left(h_{1}, h_{2}\right) \in \mathcal{H}^{\sigma+1}\left(\mathbb{T}^{2}\right)$, the transport of $\mu_{s}$ under the shift

$$
(u, v) \longmapsto(u, v)+\left(h_{1}, h_{2}\right)
$$

is singular with respect to the original measure $\mu_{s}$. Therefore, the results in Theorems 1.1 and 1.2 represent remarkable statements, displaying fine properties of the vector fields generating $\Phi_{\mathrm{NLW}}(t)$ and $\Phi_{\mathrm{NLKG}}(t)$. Moreover, we believe that the results of Theorems 1.1 and 1.2 are completely out of reach of Ramer's result 22 for which we would need $(2+\varepsilon)$ smoothing on the nonlinear term. See [27, 18] for further discussion on this topic.

According to [2], Gel'fand asked whether, in the context of Gibbs measures for Hamiltonian PDEs, one may show the quasi-invariance of the corresponding Wiener measure by a direct method. Our result gives some light on Gel'fand's question because now we have a method to directly prove quasi-invariance of a large class of Gaussian measures supported by functions of varying regularities for the nonlinear wave equations. We should also admit 
that our present understanding of the corresponding question for the (more complicated) nonlinear Schrödinger equations is quite poor.

Our main results state that the transported measure $\mu_{s}^{t}:=\Phi_{\mathrm{NLW}}(t)_{*} \mu_{s}$ by $\Phi_{\mathrm{NLW}}(t)$ (or $\Phi_{\mathrm{NLKG}}(t)$ ) is absolutely continuous with respect to $\mu_{s}$. Therefore, it has a well defined Radon-Nikodym derivative $f(t, u, v):=\frac{d \mu_{s}^{t}}{d \mu_{s}}(u, v) \in L^{1}\left(d \mu_{s}\right)$. It would be very interesting to obtain some further properties of the densities $f(t, u, v)$. We believe that a combination of our analysis and the argument in [4, Corollaire 2.2 on p. 197] leads to a higher integrability of the Radon-Nikodym derivative: $f(t, u, v) \in L^{p}\left(d \mu_{s}(u, v)\right), p<\infty$. See also Corollary 1.4 below, where the $L^{2}$-integrability of the Radon-Nikodym derivative is involved. It also seems of interest to establish some compactness properties in $t$ of $f(t, u, v)$ and to study the time averages of $f(t, u, v)$.

One of the consequences of our quasi-invariance results is the following probabilistic persistence of additional regularity (= integrability) of the solution. Let $(u(0), v(0))$ be initial data distributed according to the Gaussian measure $\mu_{s}$. Then, it follows from the Gaussian nature of the initial data that $(u(0), v(0))$ belongs to any Sobolev spaces $W^{\sigma, p}\left(\mathbb{T}^{2}\right) \times W^{\sigma-1, p}\left(\mathbb{T}^{2}\right), p \leq \infty$, and also to Hölder spaces $\mathcal{C}^{\sigma}\left(\mathbb{T}^{2}\right) \times \mathcal{C}^{\sigma-1}\left(\mathbb{T}^{2}\right)$, where $\mathcal{C}^{\sigma}\left(\mathbb{T}^{2}\right)=B_{\infty, \infty}^{\sigma}\left(\mathbb{T}^{2}\right)$, provided that $\sigma<s$. The quasi-invariance of $\mu_{s}$ guarantees the additional regularity of the global solution $(u(t), v(t))$ in the sense that, for any $t \in \mathbb{R}$, the solution $(u(t), v(t))$ almost surely belongs to the same Sobolev and Hölder spaces. Such propagation of Sobolev and Hölder regularities for general dispersive PDEs seems to be beyond deterministic analysis at this point.

We conclude this subsection by pointing out a connection of our quasi-invariance results with wave turbulence theory [31, 12]. The main goal of wave turbulence theory is to obtain a statistical description of the out-of-equilibrium dynamics given by a nonlinear dispersive PDE (for an unknown function $u(x, t)$ ). Here, randomness enters through the initial data $u(0)$ whose Fourier coefficients $\left\{\widehat{u}_{n}(0)\right\}_{n \in \mathbb{Z}^{d}}$, are assumed to be independent complex-valued Gaussian random variables with mean zero and some variance (depending on $n$, often of the form $\langle n\rangle^{-\alpha}$ ). Then, by introducing the following two-point function, 5

$$
N(n, t)=\mathbb{E}\left[\left|\widehat{u}_{n}(t)\right|^{2}\right],
$$

one aims to derive an effective closed system of equations (called the kinetic equations) for the evolution of $\{N(n, t)\}_{n \in \mathbb{Z}^{d}}$ and study its stationary solutions. Note that the twopoint functions represent the spectral density of the random field $u(t)$ and hence the kinetic equations provide evolution equations for this spectral density.

Now, let us make a connection between the study of the two-point functions (1.13) in wave turbulence theory and our quasi-invariance results. In the following, we work in a general setting, which applies to the situation in our previous works [27, 18, 14, 17] and also in this paper. For simplicity of the presentation, we consider the scalar case. Namely, let $\mu=\nu_{s}$ be the Gaussian measure defined in (1.10) and consider a nonlinear dispersive PDE on $\mathbb{T}^{d}$ for a scalar function $u$ (such as (1.9) ) with random initial data $u(0)=\varphi$ distributed

\footnotetext{
${ }^{5}$ We point out that if both the underlying equation and the distribution of $u(0)$ are translation invariant (in space), then we have

$$
\mathbb{E}\left[\widehat{u}_{n}(t) \overline{\widehat{u}_{m}(t)}\right]=0
$$

for any $t \in \mathbb{R}$, unless $n=m$. Namely, the initial uncorrelation at time 0 propagates for all times in the translation invariant setting.
} 
by $\mu$. In particular, we have

$$
\varphi(x)=\sum_{n \in \mathbb{Z}^{d}} \frac{\mathfrak{g}_{n}(\omega)}{\langle n\rangle^{s}} e^{i n \cdot x},
$$

where $\left\{\mathfrak{g}_{n}\right\}_{n \in \mathbb{Z}^{d}}$ is a sequence of independent 6 standard complex-valued Gaussian random variables on a probability space $(\Omega, \mathcal{F}, P)$. We assume that solutions exist globally in time and hence the solution map $\Phi(t): u(0)=\varphi \mapsto u(t)$ is well defined. Furthermore, we assume that the Gaussian measure $\mu$ is quasi-invariant under $\Phi(t)$. Note that this is precisely the situation in [27, 18, 14, 17].

Remark 1.3. In the setting of this paper, we need to transform the vector-valued solution $(u, v)$ to NLW (1.2) or NLKG (1.8) into a scalar (complex-valued) function $w=\frac{1}{\sqrt{2}} u+\frac{i}{\sqrt{2}}\langle\nabla\rangle^{-1} v$. If $(u(0), v(0))$ is distributed according to the Gaussian measure $\mu_{s}$ in (1.4), namely they are given by the random Fourier series in (1.5), then, by setting $\mathfrak{g}_{n}=\langle n\rangle^{s+1} \widehat{w}_{n}(0), n \in \mathbb{Z}^{2}$, we see that $\left\{\mathfrak{g}_{n}\right\}_{n \in \mathbb{Z}^{2}}$ forms a sequence of independent standard complex-valued Gaussian random variables. Hence, $w(0)=\varphi$ is distributed according to the Gaussian measure $\mu=\nu_{s+1}$ and $\varphi$ is given by the random Fourier series in (1.14) (with $s$ replaced by $s+1)$. Indeed, independence of $\mathfrak{g}_{n}$ and $\mathfrak{g}_{-n}, n \neq 0$, can be seen by writing them as

$$
\begin{aligned}
\mathfrak{g}_{n} & =\frac{\operatorname{Re} g_{n}-\operatorname{Im} h_{n}}{\sqrt{2}}+i \frac{\operatorname{Im} g_{n}+\operatorname{Re} h_{n}}{\sqrt{2}}, \\
\mathfrak{g}_{-n} & =\frac{\operatorname{Re} g_{n}+\operatorname{Im} h_{n}}{\sqrt{2}}+i \frac{-\operatorname{Im} g_{n}+\operatorname{Re} h_{n}}{\sqrt{2}},
\end{aligned}
$$

where we used $g_{-n}=\overline{g_{n}}$ and $h_{-n}=\overline{h_{n}}$. Therefore, Theorems 1.1 and 1.2 imply that, for $s \in 2 \mathbb{N}$, the Gaussian measure $\mu=\nu_{s+1}$ is quasi-invariant under the dynamics of $w(t)=\Phi(t) w(0)$. Here, the solution map $\Phi(t)$ for $w$ is given by

$$
\Phi(t)(w(0)):=\frac{1}{\sqrt{2}} \Phi_{1}(t)(u(0))+\frac{i}{\sqrt{2}}\langle\nabla\rangle^{-1} \Phi_{2}(t)(v(0)),
$$

where $\Phi_{1}(t)$ and $\Phi_{2}(t)$ denote the first and second components of the (vector-valued) solution map $\Phi_{\mathrm{NLW}}(t)$ or $\Phi_{\mathrm{NLKG}}(t)$.

Under the assumptions above, we state the following corollary to our quasi-invariance results in the general setting. This corollary allows us to express the two-point functions in terms of the Radon-Nikodym derivative.

Corollary 1.4. Let $\mu$ be the quasi-invariant measure under $\Phi(t)$ as above. We denote by $\mu^{t}=\Phi(t)_{*} \mu$ the pushforward of $\mu$ under $\Phi(t)$ and by $\frac{d \mu^{t}}{d \mu}$ its Radon-Nikodym derivative. Suppose that $\frac{d \mu^{t}}{d \mu} \in L^{2}(d \mu)$ for some $t \in \mathbb{R}$. Then, we have

$$
N(n, t)=\int|\widehat{\varphi}(n)|^{2} \frac{d \mu^{t}}{d \mu}(\varphi) d \mu(\varphi)
$$

for any $n \in \mathbb{Z}^{d}$, where $N(n, t)$ is the two-point function defined in (1.13).

\footnotetext{
${ }^{6}$ In the real-valued setting, we need to impose $\mathfrak{g}_{-n}=\overline{\mathfrak{g}_{n}}$ as in (1.5). See 27] for example.
} 
Corollary 1.4 reduces the study of the two-point functions $\{N(n, t)\}_{n \in \mathbb{Z}^{d}}$ in wave turbulence theory to studying the dynamical property of the Radon-Nikodym derivative $\frac{d \mu^{t}}{d \mu}$. This shows the importance of establishing the quasi-invariance property of the Gaussian measures from the viewpoint of wave turbulence theory. It also shows the importance of establishing a higher moment bound on the Radon-Nikodym derivative. Furthermore, by viewing $\varphi$ as

$$
\varphi: \omega \in \Omega \mapsto \varphi^{\omega}=\sum_{n \in \mathbb{Z}^{d}} \frac{\mathfrak{g}_{n}(\omega)}{\langle n\rangle^{s}} e^{i n \cdot x}
$$

we can rewrite (1.15) as

$$
N(n, t)=\int_{\Omega} \frac{\left|\mathfrak{g}_{n}(\omega)\right|^{2}-1}{\langle n\rangle^{2 s}} \frac{d \mu^{t}}{d \mu}(\varphi(\omega)) P(d \omega)+\frac{1}{\langle n\rangle^{2 s}},
$$

since $\mu=P \circ \varphi^{-1}$ by definition. Hence, it suffices to study the projection of the RadonNikodym derivative $\frac{d \mu^{t}}{d \mu}$ onto the subclass of the Wiener homogeneous chaoses of order two spanned by $\left\{\left|\mathfrak{g}_{n}\right|^{2}-1\right\}_{n \in \mathbb{Z}^{d}}$. See also Remark 1.5.

Proof of Corollary 1.4. By the definition of $\mu^{t}=\Phi(t)_{*} \mu$, we have

$$
\mu^{t}(A)=\mu(\Phi(-t) A)=\int \mathbf{1}_{\{\Phi(t) \varphi \in A\}} d \mu(\varphi)
$$

On the other hand, we have

$$
\mu^{t}(A)=\int \mathbf{1}_{\{\varphi \in A\}} d \mu^{t}(\varphi)=\int \mathbf{1}_{\{\varphi \in A\}} \frac{d \mu^{t}}{d \mu}(\varphi) d \mu(\varphi),
$$

where the existence of the Radon-Nikodym derivative $\frac{d \mu^{t}}{d \mu}$ is guaranteed by the quasiinvariance of $\mu$ under $\Phi(t)$. Hence, from (1.17) and (1.18), we obtain

$$
\int \widehat{\Phi(t) \varphi}(n) \overline{\widehat{\Phi(t) \varphi}(m)} d \mu(\varphi)=\int \widehat{\varphi}(n) \overline{\widehat{\varphi}(m)} \frac{d \mu^{t}}{d \mu}(\varphi) d \mu(\varphi) \text {. }
$$

In particular, when $n=m$, this yields (1.15).

Remark 1.5. (i) In the setting of [27, 18, 14, 17] and this paper, both the solution map $\Phi(t)$ and the Gaussian measure $\mu$ are translation invariant (in space). Hence, we have

$$
\int \widehat{\Phi(t) \varphi}(n) \overline{\widehat{\Phi(t) \varphi}(m)} d \mu(\varphi)=0
$$

for $n \neq m$. Then, it follows from (1.19) and (1.20) that

$$
\int_{\Omega} \mathfrak{g}_{n}(\omega) \overline{\mathfrak{g}_{m}(\omega)} \frac{d \mu^{t}}{d \mu}(\varphi(\omega)) P(d \omega)=0
$$

for any $n \neq m$, provided that $\frac{d \mu^{t}}{d \mu} \in L^{2}(d \mu)$. This shows that the projection of the RadonNikodym derivative $\frac{d \mu^{t}}{d \mu}$ onto a particular subclass of the Wiener homogeneous chaoses of order two (i.e. the span of $\left\{\mathfrak{g}_{n} \overline{\mathfrak{g}_{m}}\right\}_{n, m \in \mathbb{Z}^{d}, n \neq m}$ ) is 0 .

(ii) If $\langle n\rangle^{-2 s}$ happens to describe an invariant power spectrum for the underlying dynamics, namely $N(n, t)$ is independent of time for any $n \in \mathbb{Z}^{d}$, then it follows from (1.16) and (1.21) 
that

$$
\int_{\Omega} \mathfrak{g}_{n}(\omega) \overline{\mathfrak{g}_{m}(\omega)} \frac{d \mu^{t}}{d \mu}(\varphi(\omega)) P(d \omega)=\delta_{n m},
$$

completely determining the (time-independent) second order coefficients of the Wiener chaos expansion of the Radon-Nikodym derivative $\frac{d \mu^{t}}{d \mu}$.

1.4. Renormalized energy. We now derive the renormalized energies associated to NLKG (1.8). As already mentioned, these renormalized energies and the related energy estimates are the main novelty of this work. Such renormalizations usually appear in the context of low regularity solutions. We find it interesting that, in our problem, even for large $s$ (very regular solutions), we are obliged to appeal to a renormalization in constructing a modified energy. The analysis of the Benjamin-Ono equation [28] is another example, where we need to use renormalizations even for regular solutions, but in a much more perturbative manner as compared to the analysis in this paper.

In the study of the transport of $\mu_{s}$ under the flow of (1.8), we pass to the limit $N \rightarrow \infty$ in the truncated model:

$$
\left\{\begin{array}{l}
\partial_{t} u=v \\
\partial_{t} v=\Delta u-u-\pi_{N}\left(\left(\pi_{N} u\right)^{3}\right),
\end{array}\right.
$$

where $\pi_{N}$ denotes the Dirichlet projector onto the frequencies $\{|n| \leq N\}$. Then, it is easy to see that the low frequency part $E\left(\pi_{N} u, \pi_{N} v\right)$ of the energy and the truncated energy:

$$
\begin{aligned}
E_{N}(u, v) & =\frac{1}{2} \int_{\mathbb{T}^{2}}\left(u^{2}+|\nabla u|^{2}+v^{2}\right) d x+\frac{1}{4} \int_{\mathbb{T}^{2}}\left(\pi_{N} u\right)^{4} d x \\
& =E\left(\pi_{N} u, \pi_{N} v\right)+\left\|\left(\pi_{N}^{\perp} u, \pi_{N}^{\perp} v\right)\right\|_{\mathcal{H}^{1}}^{2}
\end{aligned}
$$

are conserved under the flow of (1.22), where $\pi_{N}^{\perp}=\operatorname{Id}-\pi_{N}$. Therefore, as in the case of the untruncated NLKG (1.8), the Cauchy problem for (1.22) is still globally well-posed in $\mathcal{H}^{\sigma}\left(\mathbb{T}^{2}\right), \sigma \geq 1$.

Denote $\pi_{N} u$ and $\pi_{N} v$ by $u_{N}$ and $v_{N}$, respectively. Taking into account the definition (1.4) of the Gaussian measure $\mu_{s}$, it is natural to study the expression

$$
\frac{1}{2} \frac{d}{d t}\left\|\left(u_{N}(t), v_{N}(t)\right)\right\|_{\mathcal{H}^{s+1}}^{2}
$$

where $(u, v)$ is a solution to the truncated NLKG (1.22). A direct computation yields

$$
\begin{aligned}
\frac{1}{2} \frac{d}{d t}\left\|\left(u_{N}(t), v_{N}(t)\right)\right\|_{\mathcal{H}^{s+1}}^{2} & =\partial_{t}\left[\frac{1}{2} \int_{\mathbb{T}^{2}}\left(J^{s} v_{N}\right)^{2}+\frac{1}{2} \int_{\mathbb{T}^{2}}\left(J^{s+1} u_{N}\right)^{2}\right] \\
& =\int_{\mathbb{T}^{2}}\left(J^{2 s} v_{N}\right)\left(-u_{N}^{3}\right),
\end{aligned}
$$

where

$$
J:=\sqrt{1-\Delta}
$$

In particular, when $s=0$, the term on the right-hand side is

$$
-\frac{1}{4} \partial_{t}\left[\int_{\mathbb{T}^{2}} u_{N}^{4}\right]
$$

and thus we recover the conservation of (the low frequency part of) the energy $E\left(u_{N}, v_{N}\right)$. 
Let $s \geq 2$ be an even integer. By the Leibniz rule, we have

$$
\begin{aligned}
\int_{\mathbb{T}^{2}}\left(J^{2 s} v_{N}\right)\left(-u_{N}^{3}\right)= & -3 \int_{\mathbb{T}^{2}} J^{s} v_{N} J^{s} u_{N} u_{N}^{2} \\
& +\sum_{\substack{|\alpha|+|\beta|+|\gamma| \leq s \\
|\alpha|,|\beta|,|\gamma|<s}} c_{\alpha, \beta, \gamma} \int_{\mathbb{T}^{2}} J^{s} v_{N} \cdot \partial^{\alpha} u_{N} \cdot \partial^{\beta} u_{N} \cdot \partial^{\gamma} u_{N}
\end{aligned}
$$

for some inessential constants $c_{\alpha, \beta, \gamma}$. Furthermore, recalling that $\operatorname{vol}\left(\mathbb{T}^{2}\right)=1$, we can write

$$
\begin{aligned}
-3 \int_{\mathbb{T}^{2}} J^{s} v_{N} J^{s} u_{N} u_{N}^{2}=-\frac{3}{2} \partial_{t}\left[\int_{\mathbb{T}^{2}}\left(J^{s} u_{N}\right)^{2} u_{N}^{2}\right]+3 \int_{\mathbb{T}^{2}}\left(J^{s} u_{N}\right)^{2} v_{N} u_{N} \\
=-\frac{3}{2} \partial_{t}\left[\int_{\mathbb{T}^{2}} \mathbf{P}_{\neq 0}\left[\left(J^{s} u_{N}\right)^{2}\right] \mathbf{P}_{\neq 0}\left[u_{N}^{2}\right]\right]+3 \int_{\mathbb{T}^{2}} \mathbf{P}_{\neq 0}\left[\left(J^{s} u_{N}\right)^{2}\right] \mathbf{P}_{\neq 0}\left[v_{N} u_{N}\right] \\
\quad-\frac{3}{2} \partial_{t}\left[\int_{\mathbb{T}^{2}}\left(J^{s} u_{N}\right)^{2} \int_{\mathbb{T}^{2}} u_{N}^{2}\right]+3 \int_{\mathbb{T}^{2}}\left(J^{s} u_{N}\right)^{2} \int v_{N} u_{N},
\end{aligned}
$$

where $\mathbf{P}_{\neq 0}$ is the projection onto non-zero frequencies: $\mathbf{P}_{\neq 0} f:=f-\int_{\mathbb{T}^{2}} f$. Here, the last two term 7 on the right-hand side of (1.26) are problematic because, in view of (1.5), we have

$$
\sigma_{N}:=\mathbb{E}_{\mu_{s}}\left[\int_{\mathbb{T}^{2}}\left(J^{s} u_{N}\right)^{2}\right]=\sum_{\substack{n \in \mathbb{Z}^{2} \\|n| \leq N}} \frac{1}{\langle n\rangle^{2}} \sim \log N \longrightarrow \infty
$$

as $N \rightarrow \infty$ (one may also show that we have an almost sure divergence). Therefore, we need to introduce a suitable renormalization to treat the difficulty both at the level of the $\mathcal{H}^{s+1}$-energy functional and its time derivative at the same time.

With $\sigma_{N}$ defined above, we can rewrite the last two terms on the right-hand side of (1.26) as

$$
\begin{aligned}
-\frac{3}{2} \partial_{t} & {\left[\int_{\mathbb{T}^{2}}\left(J^{s} u_{N}\right)^{2} \int u_{N}^{2}\right]+3 \int_{\mathbb{T}^{2}}\left(J^{s} u_{N}\right)^{2} \int v_{N} u_{N} } \\
& =-\frac{3}{2} \partial_{t}\left[\left(\int_{\mathbb{T}^{2}}\left(J^{s} u_{N}\right)^{2}-\sigma_{N}\right) \int_{\mathbb{T}^{2}} u_{N}^{2}\right]+3\left(\int_{\mathbb{T}^{2}}\left(J^{s} u_{N}\right)^{2}-\sigma_{N}\right) \int v_{N} u_{N} .
\end{aligned}
$$

Note that the term

$$
\int_{\mathbb{T}^{2}}\left(J^{s} u_{N}\right)^{2}-\sigma_{N}
$$

is now a "good" term since, as we shall see below, we have

$$
\left\|\int_{\mathbb{T}^{2}}\left(J^{s} \pi_{N} u\right)^{2}-\sigma_{N}\right\|_{L^{p}\left(d \mu_{s}(u, v)\right)} \leq C p
$$

for any finite $p \geq 2$, where the constant $C>0$ is independent of $p$ and $N$. In view of the above discussion, it is now natural to define the renormalized energy $E_{s, N}(u, v)$ by

$$
E_{s, N}(u, v)=\frac{1}{2} \int\left(J^{s} v\right)^{2}+\frac{1}{2} \int\left(J^{s+1} u\right)^{2}+\frac{3}{2} \int\left(J^{s} \pi_{N} u\right)^{2}\left(\pi_{N} u\right)^{2}-\frac{3}{2} \sigma_{N} \int\left(\pi_{N} u\right)^{2} .
$$

\footnotetext{
${ }^{7}$ Namely, we have issues at the level of both the energy and its time derivative.
} 
By writing $E_{s, N}(u, v)$ as

$$
\begin{aligned}
E_{s, N}(u, v)= & \frac{1}{2} \int_{\mathbb{T}^{2}}\left(J^{s} v\right)^{2}+\frac{1}{2} \int_{\mathbb{T}^{2}}\left(J^{s+1} u\right)^{2}+\frac{3}{2} \int \mathbf{P}_{\neq 0}\left[\left(J^{s} u_{N}\right)^{2}\right] \mathbf{P}_{\neq 0}\left[u_{N}^{2}\right] \\
& +\frac{3}{2}\left(\int_{\mathbb{T}^{2}}\left(J^{s} u_{N}\right)^{2}-\sigma_{N}\right) \int_{\mathbb{T}^{2}} u_{N}^{2},
\end{aligned}
$$

it follows from (1.24), (1.25), (1.26), and (1.28) that, if $(u, v)$ is a solution to (1.22), then we have

$$
\begin{aligned}
\partial_{t} E_{s, N}\left(u_{N}, v_{N}\right)= & 3 \int_{\mathbb{T}^{2}} \mathbf{P}_{\neq 0}\left[\left(J^{s} u_{N}\right)^{2}\right] \mathbf{P}_{\neq 0}\left[v_{N} u_{N}\right]+3\left(\int_{\mathbb{T}^{2}}\left(J^{s} u_{N}\right)^{2}-\sigma_{N}\right) \int_{\mathbb{T}^{2}} v_{N} u_{N} \\
& +\sum_{\substack{|\alpha|+|\beta|+|\gamma| \leq s \\
|\alpha|,|\beta|,|\gamma|<s}} c_{\alpha, \beta, \gamma} \int_{\mathbb{T}^{2}} J^{s} v_{N} \cdot \partial^{\alpha} u_{N} \cdot \partial^{\beta} u_{N} \cdot \partial^{\gamma} u_{N} .
\end{aligned}
$$

Now all terms on the right-hand side of (1.31) are suitable for a perturbative analysis. Here is the precise statement.

Theorem 1.6. Let $s \geq 2$ be an even integer and let us denote by $\Phi_{N}(t)$ the flow of (1.22). Then, given $r>0$, there is a constant $C>0$ such that

$$
\left\{\left.\int_{\left\{E_{N}(u, v) \leq r\right\}}\left|\partial_{t} E_{s, N}\left(\pi_{N} \Phi_{N}(t)(u, v)\right)\right|_{t=0}\right|^{p} d \mu_{s}(u, v)\right\}^{\frac{1}{p}} \leq C p
$$

for every $p \geq 2$ and every $N \in \mathbb{N}$.

This probabilistic energy estimate on the renormalized energy $E_{s, N}$ is the main novelty of this paper. We will present the proof of Theorem [1.6 in Section 4

Remark 1.7. It is worthwhile to note that the introduction of the renormalization at the level of the energy also introduces a renormalization at the level of the time derivative of the energy. Namely, by the argument above, we renormalized both the $\mathcal{H}^{s+1}$-energy functional and its time derivative at the same time. See (1.28), (1.30), and (1.31).

Remark 1.8. Consider the following dispersion generalized NLKG:

$$
\partial_{t}^{2} u+J^{2 \beta} u+u^{3}=0
$$

for $\beta>1$. With $v=\partial_{t} u$, we can rewrite (1.32) as

$$
\left\{\begin{array}{l}
\partial_{t} u=v \\
\partial_{t} v=-J^{2 \beta} u-u^{3} .
\end{array}\right.
$$

For this equation, we define the Gaussian measure $\mu_{s}^{\beta}$ by

$$
d \mu_{s}^{\beta}=Z_{s, \beta}^{-1} e^{-\frac{1}{2} \int\left(J^{s+\beta} u\right)^{2}-\frac{1}{2} \int\left(J^{s} v\right)^{2}} d u d v .
$$

Then, a typical element $\left(u^{\omega}, v^{\omega}\right)$ is given by the following random Fourier series:

$$
u^{\omega}(x)=\sum_{n \in \mathbb{Z}^{2}} \frac{g_{n}(\omega)}{\langle n\rangle^{s+\beta}} e^{i n \cdot x} \quad \text { and } \quad v^{\omega}(x)=\sum_{n \in \mathbb{Z}^{2}} \frac{h_{n}(\omega)}{\langle n\rangle^{s}} e^{i n \cdot x},
$$


where $\left\{g_{n}\right\}_{n \in \mathbb{Z}^{2}}$ and $\left\{h_{n}\right\}_{n \in \mathbb{Z}^{2}}$ are as in (1.5). Then, it is easy to see that $\left(u^{\omega}, v^{\omega}\right)$ belongs to

$$
H^{s+\beta-1-\varepsilon}\left(\mathbb{T}^{2}\right) \times H^{s-1-\varepsilon}\left(\mathbb{T}^{2}\right)
$$

almost surely for any $\varepsilon>0$. In particular, for $\beta>1$, we have $u \in H^{s}\left(\mathbb{T}^{2}\right)$ almost surely. In fact, we have $u \in W^{s, p}\left(\mathbb{T}^{2}\right)$ for any $p \leq \infty$ almost surely. This implies that $\int_{\mathbb{T}^{2}}\left(J^{s} u\right)^{2} u^{2}<$ $\infty$ almost surely and hence there is no need to introduce a renormalized energy. See Appendix $\mathrm{A}$.

Therefore, when $\beta>1$, one can proceed as in [27] and prove quasi-invariance of $\mu_{s}^{\beta}$ under the flow of the dispersion generalized NLKG (1.32). In particular, when $\beta=2$, (1.32) corresponds to the nonlinear beam equation on $\mathbb{T}^{2}$, which is the borderline case for Ramer's argument on $\mathbb{T}^{2}$ (namely, still non-trivial). The same remark applies to the dispersion generalized NLW:

$$
\partial_{t}^{2} u+(-\Delta)^{\beta} u+u^{3}=0
$$

1.5. Organization of the remaining part of the manuscript. We complete this section by introducing some notations. In the next section, we present the well known arguments assuring the existence of well-defined dynamics in $\mathcal{H}^{\sigma}\left(\mathbb{T}^{2}\right), \sigma \geq 1$. In Section 3 , we define a weighted Gaussian measure absolutely continuous with respect to $\mu_{s}$. This weighted Gaussian measure is adapted to the renormalized energy $E_{s, N}$ and its transport with respect to the truncated NLKG dynamics $\Phi_{N}(t)$ is easier to handle. Section 4 will be devoted to the proof of Theorem 1.6. In Section 5, we employ the arguments essentially introduced in our previous works [27, 18] to complete the proof of Theorem 1.2 for NLKG. The last section is devoted to the extension of Theorem 1.2 to the case of the "usual" nonlinear wave equation (Theorem 1.1). In Appendix $\mathrm{A}$, we briefly discuss the case of the dispersion generalized NLKG.

1.6. Notation. For a multi-index $\alpha=\left(\alpha_{1}, \alpha_{2}\right) \in \mathbb{Z}_{\geq 0}^{2}$, we set $|\alpha|=\alpha_{1}+\alpha_{2}$. For a frequency $n=\left(n_{1}, n_{2}\right) \in \mathbb{Z}^{2}$, we set $|n|=\left(n_{1}^{2}+n_{2}^{2}\right)^{\frac{1}{2}}$ and $\langle n\rangle=\left(1+n_{1}^{2}+n_{2}^{2}\right)^{\frac{1}{2}}$.

Given $N \in \mathbb{N}$, we denote the projectors $\mathbf{P}_{N}$ and $\pi_{N}$ by

$$
\left(\mathbf{P}_{N} u\right)(x)=\sum_{N \leq\langle n\rangle<2 N} \widehat{u}_{n} e^{i n \cdot x}
$$

and

$$
\left(\pi_{N} u\right)(x)=\sum_{|n| \leq N} \widehat{u}_{n} e^{i n \cdot x}
$$

We also set

$$
\pi_{N}^{\perp}=\mathrm{Id}-\pi_{N}
$$

We will consider the Littlewood-Paley decomposition of the form

$$
u=\sum_{N \geq 1, \text { dyadic }} \mathbf{P}_{N} u
$$

Given $r>0$, we define $\mu_{s, N, r}$ as

$$
d \mu_{s, N, r}(u, v)=\mathbf{1}_{\left\{E_{N}(u, v) \leq r\right\}} d \mu_{s}(u, v),
$$

where $E_{N}(u, v)$ is the conserved energy for the truncated NLKG dynamics defined in (1.23). Note that we do not normalize $\mu_{s, N, r}$ to be a probability measure. We also set $\mu_{s, r}=\mu_{s, \infty, r}$. 
Given $R>0$ and $\sigma \in \mathbb{R}$, we define the ball $B_{R, \sigma} \subset \mathcal{H}^{\sigma}\left(\mathbb{T}^{2}\right)$ by

$$
B_{R, \sigma}=\left\{(u, v) \in \mathcal{H}^{\sigma}\left(\mathbb{T}^{2}\right):\|(u, v)\|_{\mathcal{H}^{\sigma}} \leq R\right\} .
$$

\section{ON THE WELl-POSEDNESS AND APPROXIMATION PROPERTY OF THE TRUNCATED NLKG DYNAMICS}

In this section, we briefly go over the well-posedness theory of the following Cauchy problem for the truncated NLKG:

$$
\left\{\begin{array}{l}
\partial_{t} u=v \\
\partial_{t} v=\Delta u-u-\pi_{N}\left(\left(\pi_{N} u\right)^{3}\right) \\
\left.(u, v)\right|_{t=0}=\left(u_{0}, v_{0}\right)
\end{array}\right.
$$

where $N \geq 1$. We also allow $N=\infty$ with the convention $\pi_{\infty}=\mathrm{Id}$. We have the following (well-known) result.

Lemma 2.1. Let $\sigma \geq 1$ and $N \in \mathbb{N} \cup\{\infty\}$. Then, the truncated $N L K G$ (2.1) is globally wellposed in $\mathcal{H}^{\sigma}\left(\mathbb{T}^{2}\right)$. Namely, given any $\left(u_{0}, v_{0}\right) \in \mathcal{H}^{\sigma}\left(\mathbb{T}^{2}\right)$, there exists a unique global solution to (2.1) in $C\left(\mathbb{R} ; \mathcal{H}^{\sigma}\left(\mathbb{T}^{2}\right)\right)$ and, moreover, the dependence on initial data is continuous. If we denote by $\Phi_{N}(t)$ the data-to-solution map at time $t$, then $\Phi_{N}(t)$ is a continuous bijection on $\mathcal{H}^{\sigma}\left(\mathbb{T}^{2}\right)$ for every $t \in \mathbb{R}$, satisfying the semigroup property:

$$
\Phi_{N}(t+\tau)=\Phi_{N}(t) \circ \Phi_{N}(\tau)
$$

for any $t, \tau \in \mathbb{R}$.

When $N=\infty$, we simply denote $\Phi_{\infty}(t)=\Phi_{\mathrm{NLKG}}(t)$ by $\Phi(t)$ in the following.

Proof. By rewriting (2.1) in the Duhamel formulation, we have

$$
(u(t), v(t))=\bar{S}(t)\left(u_{0}, v_{0}\right)+\left(F_{1}(u)(t), F_{2}(u)(t)\right),
$$

where

$$
\bar{S}(t)\left(u_{0}, v_{0}\right)=\left(S(t)\left(u_{0}, v_{0}\right), \partial_{t} S(t)\left(u_{0}, v_{0}\right)\right)
$$

with

$$
\begin{aligned}
S(t)\left(u_{0}, v_{0}\right) & =\cos (t J) u_{0}+J^{-1} \sin (t J) v_{0}, \\
\partial_{t} S(t)\left(u_{0}, v_{0}\right) & =-J \sin (t J) u_{0}+\cos (t J) v_{0},
\end{aligned}
$$

and

$$
\begin{aligned}
& F_{1}(u)(t)=-\int_{0}^{t} J^{-1} \sin ((t-\tau) J) \pi_{N}\left(\left(\pi_{N} u\right)^{3}\right)(\tau) d \tau, \\
& F_{2}(u)(t)=-\int_{0}^{t} \cos ((t-\tau) J) \pi_{N}\left(\left(\pi_{N} u\right)^{3}\right)(\tau) d \tau .
\end{aligned}
$$

By a fixed point argument with the Sobolev embedding, one can easily solve (2.2) locally in time in $C\left([-T, T] ; \mathcal{H}^{\sigma}\left(\mathbb{T}^{2}\right)\right)$ for some small $T=T\left(\left\|\left(u_{0}, v_{0}\right)\right\|_{\mathcal{H}^{1}}\right)>0$. This claim immediately follows from the boundedness (in fact, unitarity) of $\bar{S}(t)$ on $\mathcal{H}^{\sigma}\left(\mathbb{T}^{2}\right)$ for all $\sigma \in \mathbb{R}$ and

$$
\left\|\left(F_{1}(u)(t), F_{2}(u)(t)\right)\right\|_{\mathcal{H}^{\sigma}\left(\mathbb{T}^{2}\right)} \lesssim\|u\|_{H^{\sigma}\left(\mathbb{T}^{2}\right)}\|u\|_{H^{1}\left(\mathbb{T}^{2}\right)}^{2}
$$


for any $\sigma \geq 1$. The tame estimate (2.3) is a consequence of the fractional Leibniz rule:

$$
\left\|J^{\sigma-1}\left(u^{3}\right)\right\|_{L^{2}\left(\mathbb{T}^{2}\right)} \lesssim\left\|J^{\sigma-1} u\right\|_{L^{6}\left(\mathbb{T}^{2}\right)}\|u\|_{L^{6}\left(\mathbb{T}^{2}\right)}^{2}
$$

and the Sobolev embedding: $H^{1}\left(\mathbb{T}^{2}\right) \subset L^{6}\left(\mathbb{T}^{2}\right)$ and ensures that the local existence time depends only on $\left\|\left(u_{0}, v_{0}\right)\right\|_{\mathcal{H}^{1}}$. The conservation of the truncated energy $E_{N}(u, v)$ defined in (1.23) provides an a priori bound on $\|(u(t), v(t))\|_{\mathcal{H}^{1}}$, allowing us to iterate the local existence result and extend the local solutions globally in time. The flow properties are a standard consequence of the time reversibility of (2.1). This completes the proof of Lemma 2.1.

Remark 2.2. Note that Lemma 2.1 also holds in the three-dimensional case because we also have the Sobolev embedding $H^{1}\left(\mathbb{T}^{3}\right) \subset L^{6}\left(\mathbb{T}^{3}\right)$.

We also have the following approximation property of the truncated dynamics (2.1).

Lemma 2.3. Let $\sigma \geq 1, t_{0} \in \mathbb{R}$, and $K$ be a compact set in $\mathcal{H}^{\sigma}\left(\mathbb{T}^{2}\right)$. Then, for every $\varepsilon>0$, there exists $N_{0} \in \mathbb{N}$ such that

$$
\left\|\Phi(t)(u, v)-\Phi_{N}(t)(u, v)\right\|_{\mathcal{H}^{\sigma}\left(\mathbb{T}^{2}\right)}<\varepsilon
$$

for any $t \in\left[0, t_{0}\right]$, any $(u, v) \in K$, and any $N \geq N_{0}$ and hence

$$
\Phi(t)(K) \subset \Phi_{N}(t)\left(K+B_{\varepsilon, \sigma}\right) .
$$

for any $t \in\left[0, t_{0}\right]$ and any $N \geq N_{0}$.

The proof of Lemma 2.3 is based on the identity

$$
u^{3}-\pi_{N}\left(\left(\pi_{N} u\right)^{3}\right)=\pi_{N}^{\perp}\left(u^{3}\right)+\pi_{N}\left(u^{3}-\left(\pi_{N} u\right)^{3}\right)
$$

and the estimates in the proof of Lemma 2.1] In our previous works [27, 18, we presented the details of the approximation argument analogous to Lemma 2.3 and thus we omit details.

\section{Weighted Gaussian measure associated to the Renormalized energy}

In this section, we construct a weighted Gaussian measure $\rho_{s, N, r}$ associated to the renormalized energy $E_{s, N}$ introduced in Subsection 1.4. We will study its transport properties in Section 5 .

Let $r>0$ and $N \geq 1$. In view of (1.4) and (1.29), we define a weighted Gaussian measure $\rho_{s, N, r}$ by

$$
\begin{aligned}
d \rho_{s, N, r}(u, v) & =" Z_{s, N, r}^{-1} \mathbf{1}_{\left\{E_{N}(u, v) \leq r\right\}} e^{-E_{s, N}(u, v)} d u d v " \\
& =Z_{s, N, r}^{-1} \mathbf{1}_{\left\{E_{N}(u, v) \leq r\right\}} e^{-R_{s, N}\left(\pi_{N} u\right)} d \mu_{s}(u, v),
\end{aligned}
$$

where $E_{N}(u, v)$ is the conserved energy for the truncated NLKG defined in (1.23) and $R_{s, N}(u)$ is defined by

$$
R_{s, N}(u)=\frac{3}{2} \int_{\mathbb{T}^{2}}\left(J^{s} u\right)^{2} u^{2}-\frac{3}{2} \sigma_{N} \int_{\mathbb{T}^{2}} u^{2}
$$

Our goal in this section is to prove the following statement. 
Proposition 3.1. Let $s>0$ and $r>0$. Then, given $p<\infty$, there exists $C>0$ such that

$$
\left\|\mathbf{1}_{\left\{E_{N}(u, v) \leq r\right\}} e^{-R_{s, N}\left(\pi_{N} u\right)}\right\|_{L^{p}\left(d \mu_{s}(u, v)\right)} \leq C
$$

for every $N \geq 1$. Moreover, there exists $R_{s}(u) \in L^{p}\left(d \mu_{s}(u, v)\right)$ such that

$$
\lim _{N \rightarrow \infty} R_{s, N}\left(\pi_{N} u\right)=R_{s}(u) \quad \text { in } L^{p}\left(d \mu_{s}(u, v)\right)
$$

and

$$
\lim _{N \rightarrow \infty} \mathbf{1}_{\left\{E_{N}(u, v) \leq r\right\}} e^{-R_{s, N}\left(\pi_{N} u\right)}=\mathbf{1}_{\{E(u, v) \leq r\}} e^{-R_{s}(u)} \quad \text { in } L^{p}\left(d \mu_{s}(u, v)\right) .
$$

Proposition 3.1 allows us to define the limiting weighted Gaussian measure $\rho_{s, r}$ by

$$
d \rho_{s, r}(u, v)=Z_{s, r}^{-1} \mathbf{1}_{\{E(u, v) \leq r\}} e^{-R_{s}(u)} d \mu_{s}(u, v) .
$$

Moreover, we have the following 'uniform convergence' property of $\rho_{s, N, r}$ to $\rho_{s, r}$; given any $\varepsilon>0$, there exists $N_{0} \in \mathbb{N}$ such that

$$
\left|\rho_{s, r}(A)-\rho_{s, N, r}(A)\right|<\varepsilon
$$

for any $N \geq N_{0}$ and any measurable set $A \subset \mathcal{H}^{\sigma}\left(\mathbb{T}^{2}\right), \sigma<s$.

In the following, we first state several lemmas. We then present the proof of Proposition 3.1 at the end of this section. We first recall the following Wiener chaos estimate [23, Theorem I.22]. See also [24, Proposition 2.4].

Lemma 3.2. Let $\left\{\mathfrak{g}_{n}\right\}_{n \in \mathbb{N}}$ be a sequence of independent standard real-valued Gaussian random variables. Given $k \in \mathbb{N}$, let $\left\{P_{j}\right\}_{j \in \mathbb{N}}$ be a sequence of monomials in $\overline{\mathfrak{g}}=\left\{\mathfrak{g}_{n}\right\}_{n \in \mathbb{N}}$ of degree at most $k$, namely, $P_{j}=P_{j}(\overline{\mathfrak{g}})$ is of the form $P_{j}=c_{j} \prod_{i=1}^{k_{j}} \mathfrak{g}_{n_{i}}$ with $k_{j} \leq k$ and $n_{1}, \ldots, n_{k_{j}} \in \mathbb{N}$. Then, for $p \geq 2$, we have

$$
\left\|\sum_{j \in \mathbb{N}} P_{j}(\overline{\mathfrak{g}})\right\|_{L^{p}(\Omega)} \leq(p-1)^{\frac{k}{2}}\left\|\sum_{j \in \mathbb{N}} P_{j}(\overline{\mathfrak{g}})\right\|_{L^{2}(\Omega)} .
$$

This lemma is a direct corollary to the hypercontractivity of the Ornstein-Uhlenbeck semigroup due to Nelson [13]. Note that in the definition of $P_{j}$ above, we may have $n_{i}=n_{\ell}$ for $i \neq \ell$. Namely, we do not impose independence of the factors $\mathfrak{g}_{n_{i}}$ of $P_{j}$ in Lemma 3.2. In the following, we apply Lemma 3.2 to multilinear terms involving $\left\{g_{n}\right\}_{n \in \mathbb{Z}^{2}}$ and $\left\{h_{n}\right\}_{n \in \mathbb{Z}^{2}}$ in (1.5) by first expanding $g_{n}$ and $h_{n}$ into their real and imaginary parts.

We use Lemma 3.2 to prove the following two lemmas. The first lemma is a direct consequence of the linear Gaussian bound and will be used in Section 4 .

Lemma 3.3. Let $s>1$. Let $\alpha, \beta$ be multi-indices such that $|\alpha| \leq s$ and $|\beta| \leq s-1$. Then, for every $\delta>0$, there exists $C>0$ such that

$$
\begin{aligned}
\|\| \partial^{\alpha} \mathbf{P}_{M} \pi_{N} u\left\|_{L^{\infty}\left(\mathbb{T}^{2}\right)}\right\|_{L^{p}\left(d \mu_{s}(u, v)\right)} & \leq C \sqrt{p} M^{\delta}, \\
\|\| \partial^{\beta} \mathbf{P}_{M} \pi_{N} v\left\|_{L^{\infty}\left(\mathbb{T}^{2}\right)}\right\|_{L^{p}\left(d \mu_{s}(u, v)\right)} & \leq C \sqrt{p} M^{\delta},
\end{aligned}
$$

for any $p \geq 2$ and any $N, M \in \mathbb{N}$. 
Proof. In the following, we only prove (3.8) since (3.9) follows in a similar manner. Let $q \gg 1$ be such that $q>2 / \delta$. Then, by the Sobolev embedding $W^{\delta, q}\left(\mathbb{T}^{2}\right) \subset L^{\infty}\left(\mathbb{T}^{2}\right)$, it suffices to prove the bound

$$
\|\| J^{\delta} \partial^{\alpha} \mathbf{P}_{M} \pi_{N} u\left\|_{L^{q}\left(\mathbb{T}^{2}\right)}\right\|_{L^{p}\left(d \mu_{s}(u, v)\right)} \leq C \sqrt{p} M^{\delta} .
$$

Without loss of generality, assume $p \geq q$. By Minkowski's inequality, we see that it suffices to prove

$$
\|\| J^{\delta} \partial^{\alpha} \mathbf{P}_{M} \pi_{N} u\left\|_{L^{p}\left(d \mu_{s}(u, v)\right)}\right\|_{L^{q}\left(\mathbb{T}^{2}\right)} \leq C \sqrt{p} M^{\delta}
$$

Noting that

$$
\left\|J^{\delta} \partial^{\alpha} \mathbf{P}_{M} \pi_{N} u\right\|_{L^{p}\left(d \mu_{s}(u, v)\right)}=\left\|\sum_{\substack{M \leq\langle n\rangle<2 M \\|n| \leq N}} \frac{(i n)^{\alpha}\langle n\rangle^{\delta} g_{n}}{\langle n\rangle^{s+1}} e^{i n \cdot x}\right\|_{L^{p}(\Omega)},
$$

it follows from Lemma 3.2 that

$$
\begin{aligned}
\left\|\sum_{\substack{M \leq\langle n\rangle<2 M \\
|n| \leq N}} \frac{(i n)^{\alpha}\langle n\rangle^{\delta} g_{n}}{\langle n\rangle^{s+1}} e^{i n \cdot x}\right\|_{L^{p}(\Omega)} & \leq \sqrt{p}\left\|_{\substack{M \leq\langle n\rangle<2 M \\
|n| \leq N}} \frac{(i n)^{\alpha}\langle n\rangle^{\delta} g_{n}}{\langle n\rangle^{s+1}} e^{i n \cdot x}\right\|_{L^{2}(\Omega)} \\
& =\sqrt{p}\left(\sum_{\substack{M \leq\langle n\rangle<2 M \\
|n| \leq N}} \frac{|n|^{2|\alpha|}\langle n\rangle^{2 \delta}}{\langle n\rangle^{2(s+1)}}\right)^{\frac{1}{2}} \leq C \sqrt{p} M^{\delta},
\end{aligned}
$$

yielding (3.10). This completes the proof of Lemma 3.3.

Set

$$
F_{N}(u) \equiv R_{s, N}\left(\pi_{N} u\right) .
$$

The following lemma on the convergence property of $F_{N}(u)$ is inspired by the consideration in [1. Similar analysis also appears in the quantum field theory literature.

Lemma 3.4. Let $s>0$. Then, there exist $\theta>0$ and $C>0$ such that

$$
\left\|F_{N}(u)-F_{M}(u)\right\|_{L^{p}\left(d \mu_{s}(u, v)\right)} \leq C p^{2} M^{-\theta}
$$

for any $N \geq M \geq 1$ and any $p \geq 2$.

Remark 3.5. As a corollary to Lemma 3.4, we have the following tail estimate:

$$
\mu_{s}\left((u, v):\left|F_{N}(u)-F_{M}(u)\right|>\alpha\right) \leq C e^{-c M^{\frac{\theta}{2}} \alpha^{\frac{1}{2}}},
$$

which follows from Lemma 3.4 and Chebyshev's inequality. See also [25, Lemma 4.5].

Proof. Write

$$
\frac{3}{2} \int_{\mathbb{T}^{2}}\left(J^{s} \pi_{N} u\right)^{2}\left(\pi_{N} u\right)^{2}=\frac{3}{2} \sum_{\Gamma_{N}}\left\langle n_{1}\right\rangle^{s}\left\langle n_{2}\right\rangle^{s} \widehat{u}_{n_{1}} \widehat{u}_{n_{2}} \widehat{u}_{n_{3}} \widehat{u}_{n_{4}} .
$$

where $\Gamma_{N}$ is defined by

$$
\Gamma_{N}=\left\{\left(n_{1}, n_{2}, n_{3}, n_{4}\right) \in \mathbb{Z}^{8}: n_{1}+n_{2}+n_{3}+n_{4}=0,\left|n_{j}\right| \leq N\right\} .
$$


We say that we have a pair if we have $n_{j}=-n_{k}, j \neq k$ in the summation above. Under the condition $n_{1}+n_{2}+n_{3}+n_{4}=0$, we have either two pairs or no pair. We now split the summation in three cases. (i) The first contribution comes from the case

$$
\Lambda_{1}=\Gamma_{N} \cap\left\{n_{1}=-n_{2}\right\}
$$

(ii) the second contribution comes from

$$
\Lambda_{2}=\Gamma_{N} \cap\left\{n_{1}=-n_{3} \text { or } n_{1}=-n_{4} \text { but } n_{1} \neq-n_{2}\right\},
$$

and (iii) the third contribution comes from the "no pair" case:

$$
\Lambda_{3}=\Gamma_{N} \cap\left\{n_{1} \neq-n_{j}, j=2,3,4\right\} .
$$

Therefore, recalling that $\widehat{u}_{-n}={\overline{\widehat{u}_{n}}}_{\text {}}$, we have the decomposition

$$
\frac{3}{2} \int_{\mathbb{T}^{2}}\left(J^{s} \pi_{N} u\right)^{2}\left(\pi_{N} u\right)^{2}=J_{1, N}(u)+J_{2, N}(u)+J_{3, N}(u),
$$

where $J_{j, N}(u), j=1,2,3$, is the contribution to (3.12) from $\Lambda_{j}$, satisfying

$$
\begin{aligned}
& J_{1, N}(u)=\frac{3}{2}\left(\sum_{|n| \leq N}\langle n\rangle^{2 s}\left|\widehat{u}_{n}\right|^{2}\right)\left(\sum_{|n| \leq N}\left|\widehat{u}_{n}\right|^{2}\right), \\
& J_{2, N}(u)=3 \sum_{|n| \leq N}\langle n\rangle^{s}\left|\widehat{u}_{n}\right|^{2}\left(\sum_{\substack{|m| \leq N \\
m \neq n}}\langle m\rangle^{s}\left|\widehat{u}_{m}\right|^{2}\right)-\frac{3}{2} \sum_{\substack{|n| \leq N \\
n \neq 0}}\langle n\rangle^{2 s}\left|\widehat{u}_{n}\right|^{4}, \\
& J_{3, N}(u)=\frac{3}{2} \sum_{\Lambda_{3}}\left\langle n_{1}\right\rangle^{s}\left\langle n_{2}\right\rangle^{s} \widehat{u}_{n_{1}} \widehat{u}_{n_{2}} \widehat{u}_{n_{3}} \widehat{u}_{n_{4}} .
\end{aligned}
$$

Note that the first term in 3.13 corresponds to the contribution from

$$
\left\{n_{1}=-n_{j} \text { but } n_{1} \neq-n_{2}\right\}, \quad j=3,4 .
$$

We, however, needed to subtract the contribution from

$$
\left\{n_{1}=-n_{3}=-n_{4} \text { but } n_{1} \neq-n_{2}\right\},
$$

which was counted twice. This corresponds to the second term in (3.13). Note that we need the restriction $n \neq 0$ since $n_{1} \neq-n_{2}$.

Now, by setting

$$
\widetilde{J}_{1, N}(u)=J_{1, N}(u)-\frac{3}{2} \sigma_{N} \int_{\mathbb{T}^{2}}\left(\pi_{N} u\right)^{2}=\frac{3}{2}\left(\left(\sum_{|n| \leq N}\langle n\rangle^{2 s}\left|\widehat{u}_{n}\right|^{2}\right)-\sigma_{N}\right)\left(\sum_{|n| \leq N}\left|\widehat{u}_{n}\right|^{2}\right),
$$

it suffices to prove the following three estimates:

$$
\begin{aligned}
\left\|\widetilde{J}_{1, N}\left(u^{\omega}\right)-\widetilde{J}_{1, M}\left(u^{\omega}\right)\right\|_{L^{p}(\Omega)} & \lesssim p^{2} M^{-\theta} \\
\left\|J_{2, N}\left(u^{\omega}\right)-J_{2, M}\left(u^{\omega}\right)\right\|_{L^{p}(\Omega)} & \lesssim p^{2} M^{-\theta} \\
\left\|J_{3, N}\left(u^{\omega}\right)-J_{3, M}\left(u^{\omega}\right)\right\|_{L^{p}(\Omega)} & \lesssim p^{2} M^{-\theta}
\end{aligned}
$$

where $u^{\omega}$ is as in (1.5). 
With the definition (1.27) of $\sigma_{N}$, the left-hand side of (3.14) equals

$$
\begin{aligned}
\frac{3}{2} \|\left(\sum_{|n| \leq N} \frac{\left|g_{n}\right|^{2}-1}{\langle n\rangle^{2}}\right)\left(\sum_{|n| \leq N} \frac{\left|g_{n}\right|^{2}}{\langle n\rangle^{2(s+1)}}\right) & \\
& -\left(\sum_{|n| \leq M} \frac{\left|g_{n}\right|^{2}-1}{\langle n\rangle^{2}}\right)\left(\sum_{|n| \leq M} \frac{\left|g_{n}\right|^{2}}{\langle n\rangle^{2(s+1)}}\right) \|_{L^{p}(\Omega)}
\end{aligned}
$$

Then, with

$$
A_{N} B_{N}-A_{M} B_{M}=\left(A_{N}-A_{M}\right) B_{N}+A_{M}\left(B_{N}-B_{M}\right)
$$

we can estimate (3.17) by

$$
\begin{aligned}
C\left\|\left(\sum_{M<|n| \leq N} \frac{\left|g_{n}\right|^{2}-1}{\langle n\rangle^{2}}\right)\left(\sum_{|n| \leq N} \frac{\left|g_{n}\right|^{2}}{\langle n\rangle^{2(s+1)}}\right)\right\|_{L^{p}(\Omega)} \\
\quad+C\left\|\left(\sum_{|n| \leq N} \frac{\left|g_{n}\right|^{2}-1}{\langle n\rangle^{2}}\right)\left(\sum_{M<|n| \leq N} \frac{\left|g_{n}\right|^{2}}{\langle n\rangle^{2(s+1)}}\right)\right\|_{L^{p}(\Omega)}=: \mathrm{I}+\mathrm{II} .
\end{aligned}
$$

We now estimate I and II. By Hölder's inequality, Lemma 3.2, and the triangle inequality, we have

$$
\begin{aligned}
\mathrm{I} & \lesssim\left\|_{M<|n| \leq N} \frac{\left|g_{n}\right|^{2}-1}{\langle n\rangle^{2}}\right\|_{L^{2 p}(\Omega)}\left\|\sum_{|n| \leq N} \frac{\left|g_{n}\right|^{2}}{\langle n\rangle^{2(s+1)}}\right\|_{L^{2 p}(\Omega)} \\
& \lesssim p\left\|\sum_{M<|n| \leq N} \frac{\left|g_{n}\right|^{2}-1}{\langle n\rangle^{2}}\right\|_{L^{2}(\Omega)} \sum_{|n| \leq N} \frac{\left\|g_{n}\right\|_{L^{4 p}(\Omega)}^{2}}{\langle n\rangle^{2(s+1)}} .
\end{aligned}
$$

Noting that $\left\|g_{n}\right\|_{L^{4 p}(\Omega)} \lesssim \sqrt{p}$ and

$$
\mathbb{E}\left[\left(\left|g_{n}\right|^{2}-1\right)\left(\left|g_{m}\right|^{2}-1\right)\right]=0
$$

unless $n= \pm m$, we obtain

$$
\mathrm{I} \lesssim p^{2}\left(\sum_{M<|n| \leq N} \frac{1}{\langle n\rangle^{4}}\right)^{\frac{1}{2}}\left(\sum_{|n| \leq N} \frac{1}{\langle n\rangle^{2(s+1)}}\right) \lesssim p^{2} M^{-1}
$$

Next, we estimate II. Proceeding as above, we obtain

$$
\Pi \lesssim p^{2}\left(\sum_{|n| \leq N} \frac{1}{\langle n\rangle^{4}}\right)^{\frac{1}{2}}\left(\sum_{M<|n| \leq N} \frac{1}{\langle n\rangle^{2(s+1)}}\right) \lesssim p^{2} M^{-2 s} .
$$

Hence, (3.14) follows from (3.21) and (3.22) provided that $\theta \leq \min (1,2 s)$.

Let us next turn to the proof of (3.15). By the triangle inequality, $\left\|g_{n}\right\|_{L^{4 p}(\Omega)} \lesssim \sqrt{p}$, and (3.18), we have

$$
\begin{aligned}
\text { LHS of (3.15) } & \lesssim p^{2}\left\{\left(\sum_{M<|n| \leq N} \frac{1}{\langle n\rangle^{s+2}}\right)\left(\sum_{|n| \leq N} \frac{1}{\langle n\rangle^{s+2}}\right)+\sum_{M<|n| \leq N} \frac{1}{\langle n\rangle^{2 s+4}}\right\} \\
& \leq C p^{2} M^{-s},
\end{aligned}
$$

provided that $s>0$. This proves (3.15). 
Let us finally turn to (3.16). In this case, it suffices to prove

$$
\left\|\sum_{\substack{\Lambda_{3} \\ \max \left|n_{j}\right|>M}} \frac{g_{n_{1}} g_{n_{2}} g_{n_{3}} g_{n_{4}}}{\left\langle n_{1}\right\rangle\left\langle n_{2}\right\rangle\left\langle n_{3}\right\rangle^{s+1}\left\langle n_{4}\right\rangle^{s+1}}\right\|_{L^{p}(\Omega)} \lesssim p^{2} M^{-\theta} .
$$

By Lemma 3.2, the left-hand side of (3.23) is bounded by

$$
\begin{aligned}
& p^{2} \| \sum_{\substack{\Lambda_{3} \\
\max \left|n_{j}\right|>M}} \frac{g_{n_{1}} g_{n_{2}} g_{n_{3}} g_{n_{4}}}{\left\langle n_{1}\right\rangle\left\langle n_{2}\right\rangle\left\langle n_{3}\right\rangle^{s+1}\left\langle n_{4}\right\rangle^{s+1}} \|_{L^{2}(\Omega)} \\
&=p^{2} \mathbb{E}\left[\left(\sum_{\substack{\Lambda_{3} \\
\max \left|n_{j}\right|>M}} \frac{g_{n_{1}} g_{n_{2}} g_{n_{3}} g_{n_{4}}}{\left\langle n_{1}\right\rangle\left\langle n_{2}\right\rangle\left\langle n_{3}\right\rangle^{s+1}\left\langle n_{4}\right\rangle^{s+1}}\right)\right. \\
&\left.\quad \times\left(\sum_{\max \left|m_{j}\right|>M} \frac{\frac{g_{m_{1}} g_{m_{2}} g_{m_{3} g_{m_{4}}}}{\left\langle m_{1}\right\rangle\left\langle m_{2}\right\rangle\left\langle m_{3}\right\rangle^{s+1}\left\langle m_{4}\right\rangle^{s+1}}}{\left\langle m^{2}\right.}\right)\right]^{\frac{1}{2}} .
\end{aligned}
$$

Recalling that

$$
\mathbb{E}\left[g_{n}^{k} \overline{g_{m}}{ }^{\prime}\right]=\delta_{n m} \delta_{k \ell} \cdot k !
$$

(for $n, m \neq 0$ ) 8 we see that the non-zero contribution to (3.24) comes from $m_{j}=n_{\sigma(j)}$, $j=1, \ldots, 4$, for some permutation $\sigma \in S_{4}$. Hence, we have

$$
\begin{aligned}
(3.24) & \lesssim p^{2}\left[\sum_{\substack{\Gamma_{N} \\
\max \left|n_{j}\right|>M}}\left(\frac{1}{\left\langle n_{1}\right\rangle^{2}\left\langle n_{2}\right\rangle^{2}\left\langle n_{3}\right\rangle^{2(s+1)}\left\langle n_{4}\right\rangle^{2(s+1)}}+\frac{1}{\prod_{j=1}^{4}\left\langle n_{j}\right\rangle^{s+2}}\right)\right]^{\frac{1}{2}} \\
& \lesssim p^{2} M^{-1}
\end{aligned}
$$

for $s \geq 0$. Here, the second inequality in (3.25) follows from the following estimate:

$$
\begin{aligned}
\sum_{\substack{\Gamma_{N} \\
\left|n_{1}\right|>M}} \frac{1}{\left\langle n_{1}\right\rangle^{2}\left\langle n_{2}\right\rangle^{2}\left\langle n_{3}\right\rangle^{2+\varepsilon}\left\langle n_{4}\right\rangle^{2+\varepsilon}} & =\sum_{\substack{n_{1}, n_{2}, n_{3} \in \mathbb{Z}^{2} \\
\left|n_{1}\right|>M}} \frac{1}{\left\langle n_{1}\right\rangle^{2}\left\langle n_{2}\right\rangle^{2}\left\langle n_{3}\right\rangle^{2+\varepsilon}\left\langle n_{1}+n_{2}+n_{3}\right\rangle^{2+\varepsilon}} \\
& \lesssim \sum_{\substack{n_{1}, n_{2} \in \mathbb{Z}^{2} \\
\left|n_{1}\right|>M}} \frac{1}{\left\langle n_{1}\right\rangle^{2}\left\langle n_{2}\right\rangle^{2}\left\langle n_{1}+n_{2}\right\rangle^{2+\varepsilon}} \\
& \lesssim \sum_{\substack{n_{1} \in \mathbb{Z}^{2} \\
\left|n_{1}\right|>M}} \frac{1}{\left\langle n_{1}\right\rangle^{4}} \lesssim M^{-2}
\end{aligned}
$$

for any $\varepsilon>0$. This proves (3.23) and hence (3.16). This completes the proof of Lemma 3.4.

Finally, we conclude this section by presenting the proof of Proposition 3.1 .

\footnotetext{
${ }^{8}$ Recall that $g_{0}$ is real-valued and thus we have $\mathbb{E}\left[g_{0}^{2 k}\right]=\frac{(2 k) !}{2^{k} \cdot k !}$.
} 
Proof of Proposition 3.1. First, note that (3.4) follows from Lemma 3.4. Next, let us show how Lemma 3.4 implies (3.3). It suffices to show

$$
\int_{1}^{\infty} \mu_{s, N, r}\left((u, v):-F_{N}(u)>\log \lambda\right) \lambda^{p-1} d \lambda \leq C
$$

for some finite $C>0$ independent of the truncation parameter $N$. Here, $\mu_{s, N, r}$ is the Gaussian measure $\mu_{s}$ with a cutoff on the truncated energy $E_{N}(u, v)$ defined in (1.35). While $F_{N}(u)=R_{s, N}\left(\pi_{N} u\right)$ is not sign-definite, the defocusing nature of the equation plays an important role. In fact, from (3.2) and (3.11) with (1.27), we have the following logarithmic bound:

$$
-F_{N}(u) \leq \frac{3}{2} \sigma_{N} \int_{\mathbb{T}^{2}} u^{2} \leq C_{r} \log N
$$

in the support of $\mu_{s, N, r}$. In view of this logarithmic upper bound on $-F_{N}(u)$, we apply Nelson's estimate [13] to prove (3.26). See [6, 15] for analogous arguments in the context of the $\Phi_{2}^{2 k}$-theory.

We need to estimate the measure

$$
\mu_{s, N, r}\left((u, v):-F_{N}(u)>\log \lambda\right)
$$

for each given $\lambda \geq 1$. Choose $N_{0} \in \mathbb{R}$ such that

$$
\log \lambda=2 C_{r} \log N_{0}
$$

Then, it follows from (3.27) that the contribution to (3.28) is 0 when $N<N_{0}$. On the other hand, when $N \geq N_{0}$, from (3.27) and Lemma 3.4 (see Remark 3.5), we have

$$
\begin{aligned}
\mu_{s, N, r}\left((u, v):-F_{N}(u)>\log \lambda\right) & \leq \mu_{s, N, r}\left((u, v):-F_{N}(u)+F_{N_{0}}(u)>\frac{1}{2} \log \lambda\right) \\
& \leq C e^{-c(\log \lambda)^{\frac{1}{2}} N_{0}^{\frac{\theta}{2}}}=C e^{-c(\log \lambda)^{\frac{1}{2}} \lambda^{\frac{\theta}{4 C_{r}}}} .
\end{aligned}
$$

This exponential decay ensures the bound (3.26) which in turn implies (3.3).

Finally, the uniform bound (3.3) implies (3.5) by a standard argument (see 26, Remark 3.8]). More precisely, the $L^{p}$-convergence (3.5) follows from the uniform $L^{p}$-bound (3.3) and the softer convergence in measure (as a consequence of (3.4)). This completes the proof of Proposition 3.1.

Remark 3.6. Let us briefly discuss the three-dimensional case. By repeating the computation presented above, it is easy to check that Lemma 3.4 still holds with $\theta=\min \left(\frac{1}{2}, s-1\right)$, provided that $s>1$. The main issue in proving Proposition 3.1 appears in (3.27). In the three-dimensional case, we only have

$$
-F_{N}(u) \leq C_{r} N
$$

instead of the logarithmic bound (3.27). If we were to repeat the argument above, this would force us to set $N_{0} \in \mathbb{R}$ such that

$$
\log \lambda=2 C_{r} N_{0}
$$

leading to

$$
\mu_{s, N, r}\left((u, v):-F_{N}(u)>\log \lambda\right) \leq C e^{-c(\log \lambda)^{\frac{1}{2}} N_{0}^{\frac{\theta}{2}}}=C e^{-c^{\prime}(\log \lambda)^{\frac{1}{2}+\frac{\theta}{2}}} .
$$


Noting that $\theta=\frac{1}{2}$ when $s \geq \frac{3}{2}$, we see that (3.29) is not sufficient to guarantee (3.26). As in the construction of the $\Phi_{3}^{4}$-measure, one may need to introduce a further renormalization in the three-dimensional case.

Another modification appears in Lemma 3.3. In the three-dimensional case, the estimates (3.8) and (3.9) hold with $M^{\frac{1}{2}+\delta}$ (instead of $M^{\delta}$ ). This loss makes the proof of Theorem 1.6 presented in the next section break down in the three-dimensional case. For example, in (4.4) below, we would have $p N_{4}^{-1} N_{1}^{\frac{1}{2}+\delta} N_{2}^{\frac{1}{2}+\delta}$ (instead of $p N_{4}^{-1} N_{1}^{\delta} N_{2}^{\delta}$ ), which makes the computations in Case (ii) of Subsection 4.2 simply false in the three-dimensional case. See Remark 4.1

\section{Renormalized EnERGy ESTimate}

In this section, we establish the probabilistic energy estimate on the renormalized energy (Theorem 1.6). As in Subsection 1.4, let $u_{N}=\pi_{N} u$ and $v_{N}=\pi_{N} v$. Then, from (1.31), we have

$$
\left.\partial_{t} E_{s, N}\left(\pi_{N} \Phi_{N}(t)(u, v)\right)\right|_{t=0}=Q_{1}(u, v)+Q_{2}(u, v)+Q_{3}(u, v),
$$

where

$$
\begin{aligned}
Q_{1}(u, v) & =3 \int_{\mathbb{T}^{2}} \mathbf{P}_{\neq 0}\left[\left(J^{s} u_{N}\right)^{2}\right] \mathbf{P}_{\neq 0}\left[v_{N} u_{N}\right], \\
Q_{2}(u, v) & =3\left(\int_{\mathbb{T}^{2}}\left(J^{s} u_{N}\right)^{2}-\sigma_{N}\right) \int_{\mathbb{T}^{2}} v_{N} u_{N}, \\
Q_{3}(u, v) & =\sum_{\substack{|\alpha|+|\beta|+|\gamma| \leq s \\
|\alpha|,|\beta|,|\gamma|<s}} c_{\alpha, \beta, \gamma} \int_{\mathbb{T}^{2}} J^{s} v_{N} \cdot \partial^{\alpha} u_{N} \cdot \partial^{\beta} u_{N} \cdot \partial^{\gamma} u_{N},
\end{aligned}
$$

In the following, we prove

$$
\left\|Q_{j}(u, v)\right\|_{L^{p}\left(d \mu_{s, N, r}\right)} \lesssim p
$$

for $j=1,2,3$.

4.1. Estimate on $Q_{2}(u, v)$. By Cauchy-Schwarz and Cauchy's inequalities, we have

$$
\left|\int_{\mathbb{T}^{2}} v_{N} u_{N}\right| \leq\left\|u_{N}\right\|_{L^{2}}\left\|v_{N}\right\|_{L^{2}} \leq E_{N}(u, v) .
$$

Then, proceeding as in (3.19) with Lemma 3.2 and (3.20), we have

$$
\begin{aligned}
\left\|Q_{2}(u, v)\right\|_{L^{p}\left(d \mu_{s, N, r}\right)} & \leq C_{r}\left\|\int_{\mathbb{T}^{2}}\left(J^{s} \pi_{N} u\right)^{2}-\sigma_{N}\right\|_{L^{p}\left(d \mu_{s}\right)} \\
& \sim\left\|\sum_{\substack{n \in \mathbb{Z}^{2} \\
|n| \leq N}} \frac{\left|g_{n}(\omega)\right|^{2}-1}{\langle n\rangle^{2}}\right\|_{L^{p}(\Omega)} \leq p\left\|\sum_{\substack{n \in \mathbb{Z}^{2} \\
|n| \leq N}} \frac{\left|g_{n}(\omega)\right|^{2}-1}{\langle n\rangle^{2}}\right\|_{L^{2}(\Omega)} \\
& \lesssim p
\end{aligned}
$$

This proves (4.2) in this case. 
4.2. Estimate on $Q_{1}(u, v)$. By applying the Littlewood-Paley decomposition, we have

$$
Q_{1}(u, v)=\sum_{\substack{N_{1}, N_{2}, N_{3}, N_{4} \geq 1 \\ \text { dyadic }}} Q_{1}^{\mathbf{N}}(u, v),
$$

where $\mathbf{N}:=\left(N_{1}, N_{2}, N_{3}, N_{4}\right)$ and

$$
Q_{1}^{\mathbf{N}}(u, v)=3 \int_{\mathbb{T}^{2}} \mathbf{P}_{\neq 0}\left[J^{s} \mathbf{P}_{N_{1}} u_{N} \cdot J^{s} \mathbf{P}_{N_{2}} u_{N}\right] \mathbf{P}_{\neq 0}\left[\mathbf{P}_{N_{3}} v_{N} \cdot \mathbf{P}_{N_{4}} u_{N}\right] .
$$

We consider several cases according to the sizes of $N_{1}, N_{2}, N_{3}, N_{4}$.

Case (i): $N_{4} \gtrsim \max \left(N_{1}, N_{2}\right)^{\frac{1}{100}}$.

Since $\mathbf{P}_{\neq 0}$ is clearly bounded on $L^{p}\left(\mathbb{T}^{2}\right), 1 \leq p \leq \infty$, we have

$$
\begin{aligned}
\left|Q_{1}^{\mathbf{N}}(u, v)\right| & \lesssim\left\|\mathbf{P}_{\neq 0}\left[J^{s} \mathbf{P}_{N_{1}} u_{N} \cdot J^{s} \mathbf{P}_{N_{2}} u_{N}\right]\right\|_{L_{x}^{\infty}}\left\|\mathbf{P}_{\neq 0}\left[\mathbf{P}_{N_{3}} v_{N} \cdot \mathbf{P}_{N_{4}} u_{N}\right]\right\|_{L_{x}^{1}} \\
& \leq\left\|J^{s} \mathbf{P}_{N_{1}} u_{N}\right\|_{L_{x}^{\infty}}\left\|J^{s} \mathbf{P}_{N_{2}} u_{N}\right\|_{L_{x}^{\infty}}\left\|\mathbf{P}_{N_{3}} v_{N}\right\|_{L_{x}^{2}}\left\|\mathbf{P}_{N_{4}} u_{N}\right\|_{L_{x}^{2}} .
\end{aligned}
$$

Noting that

$$
\left\|\mathbf{P}_{N_{3}} v_{N}\right\|_{L_{x}^{2}}\left\|\mathbf{P}_{N_{4}} u_{N}\right\|_{L_{x}^{2}} \lesssim N_{4}^{-1} E_{N}(u, v)
$$

we have

$$
\left\|Q_{1}^{\mathbf{N}}(u, v)\right\|_{L^{p}\left(d \mu_{s, N, r}\right)} \leq C_{r} N_{4}^{-1}\|\| J^{s} \mathbf{P}_{N_{1}} u_{N}\left\|_{L_{x}^{\infty}}\right\| J^{s} \mathbf{P}_{N_{2}} u_{N}\left\|_{L_{x}^{\infty}}\right\|_{L^{p}\left(d \mu_{s}\right)}
$$

Thanks to Lemma 3.3, we have

$$
\|\| J^{s} \mathbf{P}_{N_{j}} u_{N}\left\|_{L_{x}^{\infty}}\right\|_{L^{2 p}\left(\mu_{s}\right)} \leq C_{\delta} \sqrt{p} N_{j}^{\delta}
$$

for any $\delta>0, j=1,2$. Hence, for any $\delta>0$, we have

$$
\begin{aligned}
\left\|Q_{1}^{\mathbf{N}}(u, v)\right\|_{L^{p}\left(d \mu_{s, N, r}\right)} & \leq C_{r} N_{4}^{-1}\|\| J^{s} \mathbf{P}_{N_{1}} u_{N}\left\|_{L_{x}^{\infty}}\right\| J^{s} \mathbf{P}_{N_{2}} u_{N}\left\|_{L_{x}^{\infty}}\right\|_{L^{p}\left(d \mu_{s}\right)} \\
& \lesssim p N_{4}^{-1} N_{1}^{\delta} N_{2}^{\delta} .
\end{aligned}
$$

By noting that $Q_{1}^{\mathbf{N}}(u, v)$ is not trivial only if

$$
N_{3} \lesssim N_{1}+N_{2}+N_{4}
$$

we can readily sum (4.4) over the dyadic blocks $N_{j}, j=1, \ldots, 4$. This yields (4.2) in this case.

Remark 4.1. Thanks to Case (i), we can restrict the range of $N_{4}$ in the following. This restriction: $N_{4} \ll \max \left(N_{1}, N_{2}\right)^{\frac{1}{100}}$ plays a crucial role in Case (ii) presented below. In the three-dimensional case, due to the weaker conclusion of Lemma 3.3 mentioned in Remark [3.6. we would have $p N_{4}^{-1} N_{1}^{\frac{1}{2}+\delta} N_{2}^{\frac{1}{2}+\delta}$ on the right-hand side of (4.4). In particular, the argument above allows us to conclude (4.2) under a much stronger condition: $N_{4} \gtrsim N_{1}^{\frac{1}{2}+2 \delta} N_{2}^{\frac{1}{2}+2 \delta}$, preventing us to handle the remaining case: $N_{4} \ll N_{1}^{\frac{1}{2}+2 \delta} N_{2}^{\frac{1}{2}+2 \delta}$ in the three-dimensional setting.

Case (ii): $N_{4} \ll \max \left(N_{1}, N_{2}\right)^{\frac{1}{100}}$.

In this case, we have $\max \left(N_{1}, N_{2}\right) \sim \max \left\{N_{j}, j=1, \ldots, 4\right\}$. Without loss of generality, assume $N_{2} \leq N_{1} \sim \max \left\{N_{j}, j=1, \ldots, 4\right\}$. Let $a=a(s)>0$ be sufficiently small (to be chosen later). We consider the following two cases:

$$
\text { (ii.a): } N_{3} \ll N_{1}^{1-a} \quad \text { and } \quad \text { (ii.b): } N_{3} \gtrsim N_{1}^{1-a} \text {. }
$$


- Subcase (ii.a): $N_{3} \ll N_{1}^{1-a}$.

In this case, we have $N_{1} \sim N_{2}$. By Hölder's inequality, we have

$$
\left\|\widehat{u}_{n}\right\|_{\ell_{n}^{1+}} \lesssim E_{N}(u, v)^{\frac{1}{2}} .
$$

Then, given $p \geq 2$, it follows from Young's inequality, (4.5), and Minkowski's inequality that

$$
\begin{aligned}
& \left\|Q_{1}^{\mathbf{N}}(u, v)\right\|_{L^{p}\left(d \mu_{s, N, r}\right)} \\
& \lesssim\|\| \sum_{\substack{n=n_{1}+n_{2} \\
\left|n_{j}\right| \sim N_{j}, n_{j}|\leq N, j=1,2 \\
1 \leq| n_{1}+n_{2} \mid \ll N_{1}^{1-a}}}\left\langle n_{1}\right\rangle^{s} \widehat{u}_{n_{1}}\left\langle n_{2}\right\rangle^{s} \widehat{u}_{n_{2}}\|_{\ell_{n}^{2-}} \underbrace{\left\|\widehat{v}_{n_{3}}\right\|_{\ell_{n_{3}}^{2}}\left\|\widehat{u}_{n_{4}}\right\|_{\ell_{n_{4}}^{1+}}}_{\underbrace{}_{E_{N}(u, v)}}\|_{L^{p}\left(d \mu_{s, N, r}\right)} \\
& \leq C_{r, \varepsilon}\|\| \sum_{\substack{n=n_{1}+n_{2} \\
\left|n_{j}\right| \sim N_{j},\left|n_{j}\right| \leq N, j=1,2 \\
1 \leq\left|n_{1}+n_{2}\right| \ll N_{1}^{1-a}}}\left\langle n_{1}\right\rangle^{s} \widehat{u}_{n_{1}}\left\langle n_{2}\right\rangle^{s} \widehat{u}_{n_{2}}\left\|_{\ell_{n}^{2-\varepsilon}}\right\|_{L^{p}\left(d \mu_{s}\right)} \\
& \leq C_{r, \varepsilon}\|\|_{\substack{n=n_{1}+n_{2} \\
\left|n_{j}\right| \sim N_{j},\left|n_{j}\right| \leq N, j=1,2 \\
1 \leq\left|n_{1}+n_{2}\right| \ll N_{1}^{1-a}}}\left\langle n_{1}\right\rangle^{s} \widehat{u}_{n_{1}}\left\langle n_{2}\right\rangle^{s} \widehat{u}_{n_{2}}\left\|_{L^{p}\left(d \mu_{s}\right)}\right\|_{\ell^{2-\varepsilon}\left(|n| \lesssim N_{1}^{1-a}\right)}
\end{aligned}
$$

for any small $\varepsilon>0$. Here, we have $n_{1}+n_{2} \neq 0$ thanks to the first projection $\mathbf{P}_{\neq 0}$ in the definition (4.1) of $Q_{1}(u, v)$, while we have $\left|n_{1}+n_{2}\right|=\left|n_{3}+n_{4}\right| \lesssim \max \left(N_{3}, N_{4}\right) \ll N_{1}^{1-a}$. By the Wiener chaos estimate (Lemma 3.2) with (1.5) and $N_{1} \sim N_{2}$, we have

$$
\begin{aligned}
& \left\|Q_{1}^{\mathbf{N}}(u, v)\right\|_{L^{p}\left(d \mu_{s, N, r}\right)} \leq C_{r, \varepsilon} p\left\|\sum_{\substack{n=n_{1}+n_{2} \\
\left|n_{j}\right| \sim N_{j},\left|n_{j}\right| \leq N, j=1,2 \\
1 \leq\left|n_{1}+n_{2}\right| \ll N_{1}^{1-a}}} \frac{g_{n_{1}}(\omega)}{\left\langle n_{1}\right\rangle} \frac{g_{n_{2}}(\omega)}{\left\langle n_{2}\right\rangle}\right\|_{L^{2}(\Omega)} \|_{\ell_{n}^{2-\varepsilon}\left(|n| \lesssim N_{1}^{1-a}\right)} \\
& \lesssim C_{r, \varepsilon} p\left\|\left(\sum_{\left|n_{1}\right| \sim N_{1}} N_{1}^{-4}\right)^{\frac{1}{2}}\right\|_{\ell_{n}^{2-\varepsilon}\left(|n| \lesssim N_{1}^{1-a}\right)} \\
& \sim p N_{1}^{-1}\left\|\mathbf{1}_{|n| \lesssim N_{1}^{1-a}}\right\|_{\ell_{n}^{2-\varepsilon}} \sim p N_{1}^{-1} N_{1}^{\frac{2-2 a}{2-\varepsilon}}=p N_{1}^{\frac{-2 a+\varepsilon}{2-\varepsilon}} \text {. }
\end{aligned}
$$

Therefore, by choosing sufficiently small $\varepsilon>0$ such that $\varepsilon<2 a$, we have a negative power of $N_{1}$ that can be used to sum over the dyadic blocks. This proves (4.2) in this case.

- Subcase (ii.b): $N_{3} \gtrsim N_{1}^{1-a}$.

By Young's inequality, (4.5), and Hölder's inequality, we have

$$
\begin{aligned}
& \left\|Q_{1}^{\mathbf{N}}(u, v)\right\|_{L^{p}\left(d \mu_{s, N, r}\right)} \lesssim\left\|\sum_{\substack{n=n_{1}+n_{2}+n_{3} \\
\left|n_{j}\right| \sim N_{j},\left|n_{j}\right| \leq N, j=1,2,3 \\
n_{1}+n_{2} \neq 0}}\left\langle n_{1}\right\rangle^{s} \widehat{u}_{n_{1}}\left\langle n_{2}\right\rangle^{s} \widehat{u}_{n_{2}} \widehat{v}_{n_{3}}\right\|_{\ell_{n}^{q}} \underbrace{\left\|\widehat{u}_{n_{4}}\right\|_{\ell_{n_{4}}^{1+}}}_{\underbrace{}_{\lesssim E_{N}(u, v)^{\frac{1}{2}}}} \|_{L^{p}\left(d \mu_{s, N, r}\right)} \\
& \left|n_{1}+n_{2}+n_{3}\right| \ll N_{1}^{\frac{1}{100}}
\end{aligned}
$$




$$
\lesssim C_{r}\left\|\mathbf{1}_{\left\{E_{N}(u, v) \leq r\right\}}\right\| \sum_{\substack{n=n_{1}+n_{2}+n_{3} \\\left|n_{j}\right| \sim N_{j},\left|n_{j}\right| \leq N, j=1,2,3 \\ n_{1}+n_{2} \neq 0 \\\left|n_{1}+n_{2}+n_{3}\right| \ll N_{1} \frac{1}{100}}}\left\langle n_{1}\right\rangle^{s} \widehat{u}_{n_{1}}\left\langle n_{2}\right\rangle^{s} \widehat{u}_{n_{2}} \widehat{v}_{n_{3}}\left\|_{\ell_{n}^{q}}\right\|_{L^{p}\left(d \mu_{s}\right)}
$$

for some $q \gg 1$ (to be chosen later). Now, we can trivially write

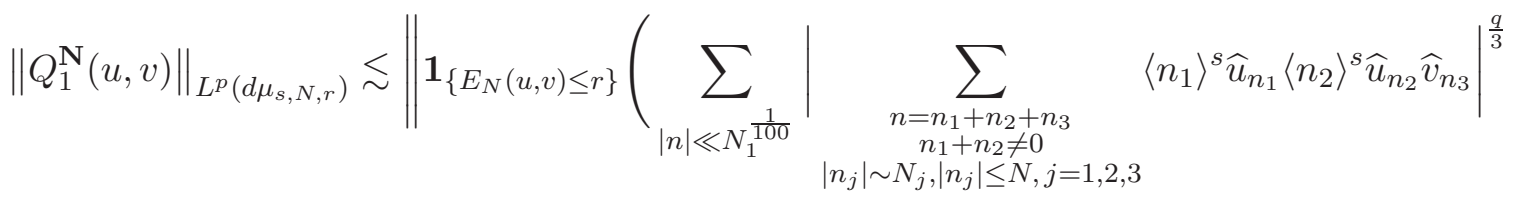

$$
\begin{aligned}
& \left.\times\left|\sum_{\substack{n=n_{1}+n_{2}+n_{3} \\
n_{1}+n_{2} \neq 0 \\
\left|n_{j}\right| \sim N_{j},\left|n_{j}\right| \leq N, j=1,2,3}}\left\langle n_{1}\right\rangle^{s} \widehat{u}_{n_{1}}\left\langle n_{2}\right\rangle^{s} \widehat{u}_{n_{2}} \widehat{v}_{n_{3}}\right|^{\frac{2 q}{3}}\right)^{\frac{1}{q}} \|_{L^{p}\left(d \mu_{s}\right)} .
\end{aligned}
$$

In the following, we estimate the first and second factors on the right-hand side above in a different manner. For the first factor, we shall use the energy restriction $E_{N}(u, v) \leq r$, while, for the second factor, we shall invoke the Wiener chaos estimate (Lemma 3.2). The balance between the powers is chosen so that we obtain $p$ to power one at the end. The main point in this procedure is that we get tractable bounds with respect to the dyadic frequency localization. Consequently, in the case under consideration, we have

$$
\begin{aligned}
& \left\|Q_{1}^{\mathbf{N}}(u, v)\right\|_{L^{p}\left(d \mu_{s, N, r}\right)} \\
& \lesssim \| \mathbf{1}_{\left\{E_{N}(u, v) \leq r\right\}}\left(\sum_{|n| \ll N_{1}^{\frac{1}{100}}}(N_{1}^{2 s-2}\left\|\left\langle n_{1}\right\rangle \widehat{u}_{n_{1}}\right\|_{\ell_{n_{1}}^{2}}\left\|\left\langle n_{2}\right\rangle \widehat{u}_{n_{2}}\right\|_{\ell_{n_{2}}^{2}} \underbrace{\left\|\widehat{v}_{n_{3}}\right\|_{\ell_{n_{3}}^{1}}}_{\lesssim N_{1}\|v\|_{L^{2}}})^{\frac{q}{3}}\right. \\
& \left.\times\left|\sum_{\substack{n=n_{1}+n_{2}+n_{3} \\
n_{1}+n_{2} \neq 0}}\left\langle n_{1}\right\rangle^{s} \widehat{u}_{n_{1}}\left\langle n_{2}\right\rangle^{s} \widehat{u}_{n_{2}} \widehat{v}_{n_{3}}\right|^{\frac{2 q}{3}}\right)^{\frac{1}{q}} \|_{L^{p}\left(d \mu_{s}\right)} \\
& \begin{array}{c}
n_{1}+n_{2} \neq 0 \\
\left|n_{j}\right| \sim N_{j},\left|n_{j}\right| \leq N, j=1,2,3
\end{array}
\end{aligned}
$$

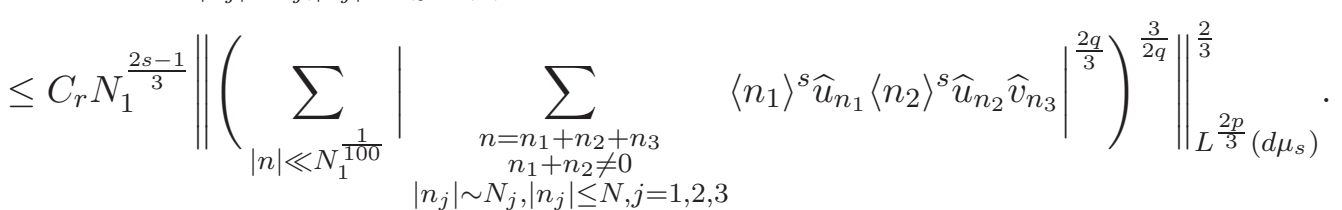

Without loss of generality, assume $p \geq q$. Then, by Minkowski's inequality and the Wiener chaos estimate (Lemma 3.2) with (1.5), we have

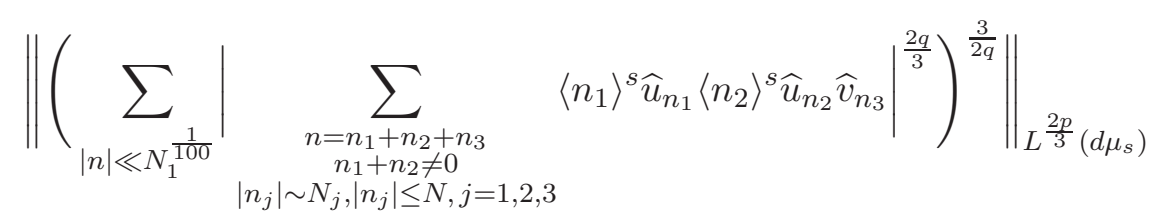




$$
\begin{aligned}
& \leq\|\| \sum_{\substack{n=n_{1}+n_{2}+n_{3} \\
n_{1}+n_{2} \neq 0 \\
\left|n_{j}\right| \sim N_{j},\left|n_{j}\right| \leq N, j=1,2,3}}\left\langle n_{1}\right\rangle^{s} \widehat{u}_{n_{1}}\left\langle n_{2}\right\rangle^{s} \widehat{u}_{n_{2}} \widehat{v}_{n_{3}}\left\|_{L^{\frac{2 p}{3}}\left(d \mu_{s}\right)}\right\|_{\ell^{\frac{2 q}{3}}\left(|n| \ll N_{1}^{\frac{1}{100}}\right)}\left\langle p^{\frac{3}{2} \|} \| \sum_{\substack{n=n_{1}+n_{2}+n_{3} \\
n_{1}+n_{2} \neq 0 \\
\left|n_{j}\right| \sim N_{j},\left|n_{j}\right| \leq N, j=1,2,3}}\left\langle\widehat{u}_{n_{1}}\left\langle n_{2}\right\rangle^{s} \widehat{u}_{n_{2}} \widehat{v}_{n_{3}}\left\|_{L^{2}\left(d \mu_{s}\right)}\right\|_{\ell^{\frac{2 q}{3}}\left(|n| \ll N_{1}^{\frac{1}{100}}\right)}\right.\right. \\
& =p^{\frac{3}{2}}\|\| \sum_{\substack{n=n_{1}+n_{2}+n_{3} \\
n_{1}+n_{2} \neq 0 \\
\left|n_{j}\right| \sim N_{j},\left|n_{j}\right| \leq N, j=1,2,3}} \frac{g_{n_{1}}}{\left\langle n_{1}\right\rangle} \frac{\left.g_{n_{2}}\right\rangle}{\left\langle n_{2}\right\rangle} \frac{h_{n_{3}}}{\left\langle n_{3}\right\rangle^{s}}\left\|_{L^{2}(\Omega)}\right\|_{\ell^{\frac{2 q}{3}}\left(|n| \ll N_{1}^{\frac{1}{100}}\right)} \\
& \left.\lesssim p^{\frac{3}{2}} \| \sum_{\substack{\left|n_{j}\right| \sim N_{j}, j=2,3}} N_{1}^{-2} \frac{1}{\left\langle n_{2}\right\rangle^{2}} \frac{1}{\left\langle n_{3}\right\rangle^{2 s}}\right)^{\frac{1}{2}} \|_{\ell_{n}^{\frac{2 q}{3}}\left(|n| \ll N_{1}^{\frac{1}{100}}\right)} \cdot
\end{aligned}
$$

Summing over $n_{2}$ and $n_{3}$ with $\left|n_{2}\right| \sim N_{2}$ and $N_{1}^{1-a} \lesssim N_{3} \lesssim N_{1}$, we have

$$
\begin{aligned}
\text { LHS of (4.7) } & \lesssim p^{\frac{3}{2}}\left\|\left(N_{1}^{-2} N_{1}^{(-2 s+2)(1-a)}\right)^{\frac{1}{2}}\right\|_{\ell_{n}^{\frac{2 q}{3}}\left(|n| \ll N_{1}^{\frac{1}{100}}\right)} \\
& =p^{\frac{3}{2}} N_{1}^{-s+a s-a}\left\|\mathbf{1}_{|n| \ll N_{1}^{\frac{1}{100}}}\right\|_{\ell_{n}^{\frac{2 q}{3}}} \lesssim p^{\frac{3}{2}} N_{1}^{-s+a s-a} N_{1}^{\frac{3}{100 q}} .
\end{aligned}
$$

Therefore, by choosing sufficiently large $q \gg 1$ and sufficiently small $a=a(s)>0$, it follows from (4.6) and (4.8) that

$$
\left\|Q_{1}^{\mathbf{N}}(u, v)\right\|_{L^{p}\left(d \mu_{s, N, r}\right)} \lesssim C p N_{1}^{-\frac{1}{3}+\frac{2}{3} a s-\frac{2}{3} a+\frac{1}{50 q}} \lesssim C p N_{1}^{-\alpha}
$$

for some $\alpha>0$. Once again, we obtained a negative power of $N_{1}$, allowing us to sum over the dyadic blocks. This proves (4.2) in Subcase (ii.b).

4.3. Estimate on $Q_{3}(u, v)$. It remains to prove (4.2) for $j=3$. It turns out that $Q_{3}(u, v)$ can be estimated essentially in the same manner as $Q_{1}(u, v)$. By integration by parts, we can express each summand in the definition of $Q_{3}(u, v)$ as

$$
\int_{\mathbb{T}^{2}} \partial^{\kappa} v_{N} \cdot \partial^{\alpha} u_{N} \cdot \partial^{\beta} u_{N} \cdot \partial^{\gamma} u_{N}
$$

where $|\kappa| \leq s-1,|\alpha|+|\beta|+|\gamma| \leq s+1$, and $\max (|\alpha|,|\beta|,|\gamma|) \leq s$.

Let us first consider the case $\max (|\alpha|,|\beta|,|\gamma|)=s$. By symmetry, we assume that $|\alpha| \geq|\beta| \geq|\gamma|$ and therefore $|\alpha|=s$. We then necessarily have $|\beta|=1$ and $|\gamma|=0$. Then, we can treat (4.9) exactly in the same manner as we did for $Q_{1}(u, v)$ by replacing $\mathbf{P}_{\neq 0}\left[\left(J^{s} u_{N}\right)^{2}\right]$ and $\mathbf{P}_{\neq 0}\left[v_{N} u_{N}\right]$ in the definition (4.1) of $Q_{1}(u, v)$ with $\partial^{\kappa} v_{N} \cdot \partial^{\alpha} u_{N}$ and $\partial^{\beta} u_{N} \cdot \partial^{\gamma} u_{N}$, respectively. Note that, while the frequency projection $\mathbf{P}_{\neq 0}\left[\left(J^{s} u_{N}\right)^{2}\right]$ in the definition of $Q_{1}(u, v)$ played an important role in eliminating the logarithmic divergence, we do not need a frequency projection $\mathbf{P}_{\neq 0}$ on $\partial^{\kappa} v_{N} \cdot \partial^{\alpha} u_{N}$ since, in view of (1.5), the independence of $v_{N}$ and $u_{N}$ prevents such logarithmic divergence. 
Therefore, we can suppose that $\max (|\alpha|,|\beta|,|\gamma|) \leq s-1$. We only consider the worst case $|\alpha|+|\beta|+|\gamma|=s+1$ and $|\kappa|=s-1$ in the following. In this case, noting that $\partial^{\kappa} v_{N}$ with $|\kappa|=s-1$ behaves like $J^{s} \pi_{N} u$ (see (1.5)), we can basically proceed as we $\operatorname{did}$ for $Q_{1}(u, v)$ in the previous subsection. Indeed, by applying the Littlewood-Paley decomposition, we need to study the expression of the form

$$
\widetilde{Q}_{1}^{\mathbf{N}}(u, v)=\int_{\mathbb{T}^{2}} \partial^{\kappa} \mathbf{P}_{N_{1}} v_{N} \cdot \partial^{\alpha} \mathbf{P}_{N_{2}} u_{N} \cdot \partial^{\beta} \mathbf{P}_{N_{3}} u_{N} \cdot \partial^{\gamma} \mathbf{P}_{N_{4}} u_{N}
$$

By symmetry, assume $N_{2} \geq N_{3} \geq N_{4}$. Then, we have

$$
\widetilde{Q}_{1}^{\mathbf{N}}(u, v) \sim \int_{\mathbb{T}^{2}} \partial^{\kappa} \mathbf{P}_{N_{1}} v_{N} \cdot N_{2}^{s-|\alpha|} \partial^{\alpha} \mathbf{P}_{N_{2}} u_{N} \cdot N_{2}^{1-|\beta|} \partial^{\beta} \mathbf{P}_{N_{3}} u_{N} \cdot N_{2}^{-|\gamma|} \partial^{\gamma} \mathbf{P}_{N_{4}} u_{N}
$$

As mentioned above, the first factor $\partial^{\kappa} \mathbf{P}_{N_{1}} v_{N}$ in (4.10) behaves like $J^{s} \mathbf{P}_{N_{1}} u_{N}$ in (4.3). The second factor $N_{2}^{s-|\alpha|} \partial^{\alpha} \mathbf{P}_{N_{2}} u_{N} \sim \partial^{\widetilde{\alpha}} \mathbf{P}_{N_{2}} u_{N}$ with $|\widetilde{\alpha}|=s$ also behaves like the second factor $J^{s} \mathbf{P}_{N_{2}} u_{N}$ in (4.3). Similarly, the third and fourth factors in (4.10):

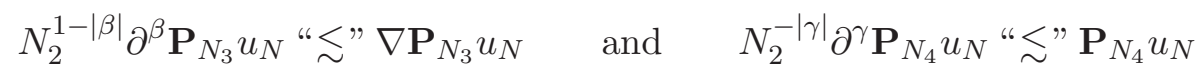

behave (at worst) like the third and fourth factors in (4.3), respectively. Hence, we can estimate $\widetilde{Q}_{1}^{\mathbf{N}}(u, v)$ just as we did for $Q_{1}^{\mathbf{N}}(u, v)$ in the previous section. This completes the proof of Theorem 1.6.

\section{Proof of Theorem 1.2}

In this section, we prove quasi-invariance of the Gaussian measure $\mu_{s}$ under the NLKG dynamics (Theorem 1.2). While the general structure of the argument is similar to our previous works [27, 18] (see also [19] for a concise sketch of the general structure), we proceed differently in some part (see Proposition 5.3).

5.1. A change-of-variable formula. As in our previous works [27, 18], the change-ofvariable formula (Lemma 5.1) for the nonlinear transformation induced by the truncated flow $\Phi_{N}(t)$ plays an important role. We also point out that these change-of-variable formulas in this paper and in [27, 18] are in turn inspired by [29].

Let $\Lambda$ be as in (1.6). Given $N \in \mathbb{N}$, we denote by $\mathcal{E}_{N}$ the real vector space:

$$
\mathcal{E}_{N}=\operatorname{span}\left\{1, \cos (n \cdot x), \sin (n \cdot x): n \in \Lambda_{N}^{*}\right\},
$$

where $\Lambda_{N}^{*}=\left\{n \in \mathbb{Z}^{2}: 0<|n| \leq N\right\} \cap \Lambda$. We equip $\mathcal{E}_{N}$ with the natural scalar product. Moreover, we endow $\mathcal{E}_{N} \times \mathcal{E}_{N}$ with a Lebesgue measure $L_{N}$ as follows. Given

$$
\left(\pi_{N} u\right)(x)=\sum_{|n| \leq N} \widehat{u}_{n} e^{i n \cdot x}, \quad \widehat{u}_{-n}={\overline{u_{n}}}_{n}
$$

let $a_{n}=\operatorname{Re} \widehat{u}_{n}$ and $b_{n}=\operatorname{Im} \widehat{u}_{n},\left(a_{n}, b_{n}\right) \in \mathbb{R}^{2}$. Then, we have

$$
\left(\pi_{N} u\right)(x)=a_{0}+\sum_{n \in \Lambda_{N}^{*}}\left\{a_{n}(2 \cos (n \cdot x))+b_{n}(-2 \sin (n \cdot x))\right\} .
$$

Therefore, it is natural to define $L_{N}$ as the Lebesgue measure on $\mathcal{E}_{N} \times \mathcal{E}_{N}$ with respect to the orthogonal basis:

$$
\left\{1,\{2 \cos (n \cdot x),-2 \sin (n \cdot x)\}_{n \in \Lambda_{N}^{*}}\right\} \times\left\{1,\{2 \cos (n \cdot x),-2 \sin (n \cdot x)\}_{n \in \Lambda_{N}^{*}}\right\} .
$$


Next, we denote by $\left(\mathcal{E}_{N} \times \mathcal{E}_{N}\right)^{\perp}$ the orthogonal complement of $\mathcal{E}_{N} \times \mathcal{E}_{N}$ in $\mathcal{H}^{\sigma}\left(\mathbb{T}^{2}\right), \sigma<s$. We endow $\left(\mathcal{E}_{N} \times \mathcal{E}_{N}\right)^{\perp}$ with the marginal Gaussian measure $\mu_{s ; N}^{\perp}$ on $\pi_{N}^{\perp} \mathcal{H}^{\sigma}\left(\mathbb{T}^{2}\right)$ which is defined as the induced probability measure under the map:

$$
\omega \in \Omega \longmapsto\left(\pi_{N}^{\perp} u^{\omega}, \pi_{N}^{\perp} v^{\omega}\right),
$$

where $\left(u^{\omega}, v^{\omega}\right)$ is as in (1.5). By viewing the Gaussian measure $\mu_{s}$ as a product measure on $\left(\mathcal{E}_{N} \times \mathcal{E}_{N}\right) \times\left(\mathcal{E}_{N} \times \mathcal{E}_{N}\right)^{\perp}$, we can write the truncated weighted Gaussian measure $\rho_{s, N, r}$ defined in (3.1) as

$$
\begin{aligned}
d \rho_{s, N, r}(u, v) & =Z_{s, N, r}^{-1} \mathbf{1}_{\left\{E_{N}(u, v) \leq r\right\}} e^{-R_{s, N}\left(\pi_{N} u\right)} d \mu_{s}(u, v) \\
& =\hat{Z}_{s, N, r}^{-1} \mathbf{1}_{\left\{E_{N}(u, v) \leq r\right\}} e^{-E_{s, N}\left(\pi_{N} u, \pi_{N} v\right)} d L_{N} \otimes d \mu_{s ; N}^{\perp},
\end{aligned}
$$

where $\hat{Z}_{s, N, r}$ is defined by

$$
\hat{Z}_{s, N, r}=\int_{\mathcal{H}^{\sigma}\left(\mathbb{T}^{2}\right)} \mathbf{1}_{\left\{E_{N}(u, v) \leq r\right\}} e^{-E_{s, N}\left(\pi_{N} u, \pi_{N} v\right)} d L_{N} \otimes d \mu_{s ; N}^{\perp} .
$$

Then, we have the following change-of-variable formula.

Lemma 5.1. Let $s>1, N \in \mathbb{N}$, and $r>0$. Then, we have

$$
\rho_{s, N, r}\left(\Phi_{N}(t)(A)\right)=\hat{Z}_{s, N, r}^{-1} \int_{A} \mathbf{1}_{\left\{E_{N}(u, v) \leq r\right\}} e^{-E_{s, N}\left(\pi_{N} \Phi_{N}(t)(u, v)\right)} d L_{N} \otimes d \mu_{s ; N}^{\perp}
$$

for any $t \in \mathbb{R}$ and any measurable set $A \subset \mathcal{H}^{\sigma}\left(\mathbb{T}^{2}\right), \sigma \in(1, s)$.

Lemma 5.1 follows from similar considerations presented in [27, 18] and therefore we omit its proof.

5.2. The evolution of the truncated measures. We now study the evolution of the truncated measures $\rho_{s, N, r}$. We shall use the renormalized energy estimate (Theorem 1.6) as a key step in the proof of the following statement. Due to the use of Theorem 1.6, we assume that $s \geq 2$ is an even integer in the following. While all the implicit constants depend on $s$, we may not state their dependence in an explicit manner.

Lemma 5.2. Given $r>0$, there exists $C_{r}>0$ such that

$$
\frac{d}{d t} \rho_{s, N, r}\left(\Phi_{N}(t)(A)\right) \leq C_{r} p\left\{\rho_{s, N, r}\left(\Phi_{N}(t)(A)\right)\right\}^{1-\frac{1}{p}}
$$

for any $p \geq 2$, any $N \in \mathbb{N}$, any $t \in \mathbb{R}$, and any measurable set $A \subset \mathcal{H}^{\sigma}\left(\mathbb{T}^{2}\right), \sigma \in(1, s)$.

While the proof of Lemma 5.2 also follows from the argument in our previous works [27, 18, we present its details in order to show the use of the crucial renormalized energy estimate.

Proof. Fix $t_{0} \in \mathbb{R}$. As in [29, 27, 18], the main idea is to reduce the analysis to that at $t=0$. Using the flow property of $\Phi_{N}(t)$, we have

$$
\begin{aligned}
\left.\frac{d}{d t} \rho_{s, N, r}\left(\Phi_{N}(t)(A)\right)\right|_{t=t_{0}} & =\left.Z_{s, N, r}^{-1} \frac{d}{d t} \int_{\Phi_{N}(t)(A)} \mathbf{1}_{\left\{E_{N}(u, v) \leq r\right\}} e^{-R_{s, N}\left(\pi_{N} u\right)} d \mu_{s}(u, v)\right|_{t=t_{0}} \\
& =\left.Z_{s, N, r}^{-1} \frac{d}{d t} \int_{\Phi_{N}(t)\left(\Phi_{N}\left(t_{0}\right)(A)\right)} \mathbf{1}_{\left\{E_{N}(u, v) \leq r\right\}} e^{-R_{s, N}\left(\pi_{N} u\right)} d \mu_{s}(u, v)\right|_{t=0} .
\end{aligned}
$$


By the change-of-variable formula (Lemma 5.1), we have

$$
\begin{aligned}
& \left.\frac{d}{d t} \rho_{s, N, r}\left(\Phi_{N}(t)(A)\right)\right|_{t=t_{0}} \\
& \quad=\left.\hat{Z}_{s, N, r}^{-1} \frac{d}{d t} \int_{\Phi_{N}\left(t_{0}\right)(A)} \mathbf{1}_{\left\{E_{N}(u, v) \leq r\right\}} e^{-E_{s, N}\left(\pi_{N} \Phi_{N}(t)(u, v)\right)} d L_{N} \otimes d \mu_{s ; N}^{\perp}\right|_{t=0} \\
& \quad=\left.Z_{s, N, r}^{-1} \int_{\Phi_{N}\left(t_{0}\right)(A)} \mathbf{1}_{\left\{E_{N}(u, v) \leq r\right\}} \partial_{t} E_{s, N}\left(\pi_{N} \Phi_{N}(t)(u, v)\right)\right|_{t=0} e^{-R_{s, N}\left(\pi_{N} u\right)} d \mu_{s}(u, v) .
\end{aligned}
$$

Now, Hölder's inequality yields

$$
\begin{aligned}
\left.\frac{d}{d t} \rho_{s, N, r}\left(\Phi_{N}(t)(A)\right)\right|_{t=t_{0}} \leq\left\|\left.\partial_{t} E_{s, N}\left(\pi_{N} \Phi_{N}(t)(u, v)\right)\right|_{t=0}\right\|_{L^{p}\left(\rho_{s, N, r}\right)} & \times\left\{\rho_{s, N, r}\left(\Phi_{N}\left(t_{0}\right)(A)\right)\right\}^{1-\frac{1}{p}}
\end{aligned}
$$

Observe that Proposition 3.1 implies that $Z_{s, N, r}^{-1}$ is bounded, uniformly in $N$. Finally, by Cauchy-Schwarz inequality together with the uniform estimate (3.3) in Proposition 3.1 and Theorem 1.6, we obtain

$$
\begin{aligned}
\| \partial_{t} E_{s, N} & \left.\left(\pi_{N} \Phi_{N}(t)(u, v)\right)\right|_{t=0} \|_{L^{p}\left(\rho_{s, N, r}\right)} \\
& \leq Z_{s, N, r}^{-\frac{1}{p}}\left\|\left.\partial_{t} E_{s, N}\left(\pi_{N} \Phi_{N}(t)(u, v)\right)\right|_{t=0}\right\|_{L^{2 p}\left(\mu_{s, N, r}\right)}\left\|\mathbf{1}_{\left\{E_{N}(u, v) \leq r\right\}} e^{-R_{s, N}\left(\pi_{N} u\right)}\right\|_{L^{2}\left(\mu_{s}\right)}^{\frac{1}{p}} \\
& \leq C_{r} p
\end{aligned}
$$

since $Z_{s, N, r}^{-\frac{1}{p}} \leq C(s, r)$ for any $p \geq 2$ and $N \in \mathbb{N}$. This completes the proof of Lemma 5.2

As a corollary to Lemma 5.2, we obtain the following control on the truncated measures $\rho_{s, N, r}$. We point out that this is where our argument diverges from the presentation in our previous works [27, 18].

Proposition 5.3. Given $r>0$, there exists $t_{r}>0$ such that given $\varepsilon>0$, there exists $\delta>0$ such that if, for a measurable set $A \subset \mathcal{H}^{\sigma}\left(\mathbb{T}^{2}\right), \sigma \in(1, s)$, there exists $N_{0} \in \mathbb{N}$ such that

$$
\rho_{s, N, r}(A)<\delta
$$

for any $N \geq N_{0}$, then we have

$$
\rho_{s, N, r}\left(\Phi_{N}(t)(A)\right)<\varepsilon
$$

for any $t \in\left[0, t_{r}\right]$ and any $N \geq N_{0}$.

Remark 5.4. In Proposition [5.3, we can choose $t_{r}>0$ and $\delta>0$ such that they are independent of $N \in \mathbb{N}$. Moreover, $\delta>0$ is independent of $t_{r}>0$.

Proof. From Lemma 5.2, we have

$$
\frac{d}{d t}\left\{\rho_{s, N, r}\left(\Phi_{N}(t)(A)\right)\right\}^{\frac{1}{p}} \leq C_{r}
$$

for any $p \geq 2$. Integrating (5.1) from 0 to $t$, we obtain

$$
\rho_{s, N, r}\left(\Phi_{N}(t)(A)\right) \leq\left\{\left(\rho_{s, N, r}(A)\right)^{\frac{1}{p}}+C_{r} t\right\}^{p} .
$$


Now, choose $t_{r}>0$ such that $C_{r} t_{r}=\frac{1}{4}$. Without loss of generality, assume $\varepsilon<1$. It follows from (5.2) and the convexity inequality:

$$
\left(\frac{x+y}{2}\right)^{p} \leq \frac{x^{p}+y^{p}}{2}, \quad x, y \geq 0, p \geq 1
$$

that for $t \in\left[0, t_{r}\right]$,

$$
\begin{aligned}
\rho_{s, N, r}\left(\Phi_{N}(t)(A)\right) & \leq 2^{p-1} \rho_{s, N, r}(A)+2^{p-1}\left(C_{r} t_{r}\right)^{p} \\
& \leq 2^{p-1} \rho_{s, N, r}(A)+2^{-p-1}
\end{aligned}
$$

by setting $p=p(\varepsilon)=-\log _{2} \varepsilon$,

$$
\begin{aligned}
& \leq 2^{p(\varepsilon)-1} \delta+\frac{1}{2} \varepsilon \\
& <\varepsilon
\end{aligned}
$$

by choosing $\delta=\delta(\varepsilon)>0$ sufficiently small. This completes the proof of Proposition 5.3 .

5.3. Proof of Theorem 1.2. We conclude this section by presenting the proof of Theorem 1.2. Proposition 5.3 implies that the truncated weighted Gaussian measures $\rho_{s, N, r}$ are quasi-invariant under the truncated NLKG dynamics $\Phi_{N}(t)$ with the uniform control in $N \in \mathbb{N}$. We first upgrade Proposition 5.3 to the untruncated weighted Gaussian measure $\rho_{s, r}$ defined in (3.6). Then, we exploit the mutual absolute continuity between $\rho_{s, r}$ and $\mu_{s, r}$, implying quasi-invariance of $\mu_{s, r}$ under the full NLKG dynamics $\Phi(t)=\Phi_{\mathrm{NLKG}}(t)$. Finally, we conclude quasi-invariance of $\mu_{s}$ by taking $r \rightarrow \infty$.

Lemma 5.5. Given $r>0$, there exists $t_{r}>0$ such that given $\varepsilon>0$, there exists $\delta>0$ such that if

$$
\rho_{s, r}(A)<\delta
$$

for a measurable set $A \subset \mathcal{H}^{\sigma}\left(\mathbb{T}^{2}\right), \sigma \in(1, s)$, then we have

$$
\rho_{s, r}(\Phi(t)(A))<\varepsilon
$$

for any $t \in\left[0, t_{r}\right]$. Note that $\delta>0$ is independent of $t \in\left[0, t_{r}\right]$.

Proof. Let $t_{r}$ be as in Proposition 5.3. We first consider the case when $A$ is compact in $\mathcal{H}^{\sigma}\left(\mathbb{T}^{2}\right)$. Let $\varepsilon>0$. Thanks to Proposition 5.3, there is $\delta_{1}>0$ such that if there exists $N_{0} \in \mathbb{N}$ such that

$$
\rho_{s, N, r}\left(A+B_{\theta, \sigma}\right)<\delta_{1}
$$

for any $N \geq N_{0}$, then we have

$$
\rho_{s, N, r}\left(\Phi_{N}(t)\left(A+B_{\theta, \sigma}\right)\right)<\frac{\varepsilon}{2}
$$

for any $t \in\left[0, t_{r}\right]$ and any $N \geq N_{0}$. Recall that $B_{\theta, \sigma}$ denotes the (closed) ball of radius $\theta>0$ in $\mathcal{H}^{\sigma}\left(\mathbb{T}^{2}\right)$.

We now observe that there exist $\delta_{2}>0, N_{1} \in \mathbb{N}$, and $\theta>0$ such that if

$$
\rho_{s, N, r}(A)<\delta_{2}
$$

for any $N \geq N_{1}$, then we have

$$
\rho_{s, N, r}\left(A+B_{\theta, \sigma}\right)<\delta_{1}
$$


for any $N \geq N_{1}$. More precisely, by writing

$$
d \rho_{s, N, r}(u, v)=G_{N}(u, v) d \mu_{s}(u, v) \quad \text { and } \quad d \rho_{s, r}(u, v)=G(u, v) d \mu_{s}(u, v),
$$

it follows from Proposition 3.1 that $G_{N}$ converges to $G$ in $L^{p}\left(d \mu_{s}\right)$ for every $p<\infty$. We can therefore write

$$
\begin{aligned}
\rho_{s, N, r}\left(A+B_{\theta, \sigma}\right) & -\rho_{s, N, r}(A)=\int_{A+B_{\theta, \sigma}} G_{N} d \mu_{s}-\int_{A} G_{N} d \mu_{s} \\
& =\int_{A+B_{\theta, \sigma}}\left(G_{N}-G\right) d \mu_{s}+\int\left(\mathbf{1}_{A+B_{\theta, \sigma}}-\mathbf{1}_{A}\right) G d \mu_{s}+\int_{A}\left(G-G_{N}\right) d \mu_{s} .
\end{aligned}
$$

Now, for the first and third terms, we use the convergence of $G_{N}$ to $G$ in $L^{1}\left(d \mu_{s}\right)$, while, for the second term, we invoke the dominated convergence (here we used the fact that $A$ is closed). Therefore, we conclude that (5.4) implies (5.5). We also observe that thanks to (3.7), there exist $\delta>0$ and $N_{2} \in \mathbb{N}$ such that if

$$
\rho_{s, r}(A)<\delta,
$$

then we have (5.4) for any $N \geq N_{2}$. At this point, we have already fixed the values of $\delta, \theta$, $N_{0}, N_{1}$, and $N_{2}$. Finally, it follows Lemma 2.3 and (3.7) that there exists $N_{3}=N_{3}(t, \theta, \varepsilon) \in$ $\mathbb{N}$ such that if (5.6) holds, then we have

$$
\rho_{s, r}(\Phi(t)(A)) \leq \rho_{s, r}\left(\Phi_{N}(t)\left(A+B_{\theta, \sigma}\right)\right) \leq \rho_{s, N, r}\left(\Phi_{N}(t)\left(A+B_{\theta, \sigma}\right)\right)+\frac{\varepsilon}{2}<\varepsilon
$$

for any $t \in\left[0, t_{r}\right]$ and any $N \geq \max \left(N_{0}, N_{1}, N_{2}, N_{3}\right)$. Here, we used (3.7) and (5.3) in the second and third inequalities, respectively. This completes the proof when $A$ is compact.

We now prove the statement for arbitrary measurable sets. Once again, fix $\varepsilon>0$. We have just proved that there is $\delta>0$ such that, for every compact set $K$ with $\rho_{s, r}(K)<\delta$, we have

$$
\rho_{s, r}(\Phi(t)(K))<\frac{\varepsilon}{2}
$$

for any $t \in\left[0, t_{r}\right]$. Now, let $A$ be an arbitrary measurable set of $\mathcal{H}^{\sigma}\left(\mathbb{T}^{2}\right), \sigma \in(1, s)$, such that $\rho_{s, r}(A)<\delta$. By the inner regularity of $\rho_{s, r}$, there exists a sequence $\left\{K_{j}\right\}_{j \in \mathbb{N}}$ of compact sets such that $K_{j} \subset \Phi(t)(A)$ and

$$
\rho_{s, r}(\Phi(t)(A))=\lim _{j \rightarrow \infty} \rho_{s, r}\left(K_{j}\right) .
$$

Note that $\Phi(-t)\left(K_{j}\right)$ is compact since it is the image of the compact set $K_{j}$ under the continuous map $\Phi(-t)$. Moreover, by the bijectivity of the flow $\Phi(-t)$, we have $\Phi(-t)\left(K_{j}\right) \subset \Phi(-t) \Phi(t)(A)=A$. In particular, we have $\rho_{s, r}\left(\Phi(-t)\left(K_{j}\right)\right)<\delta$. Then, applying (5.7) for the compact set $\Phi(-t) K_{j}$, we obtain

$$
\rho_{s, r}\left(K_{j}\right)=\rho_{s, r}\left(\Phi(t)\left(\Phi(-t) K_{j}\right)\right)<\frac{\varepsilon}{2}
$$

for all $j \in \mathbb{N}$ and all $t \in\left[0, t_{r}\right]$. Hence, the desired conclusion follows from (5.8) and (5.9) . This completes the proof of Lemma 5.5 .

Finally, we present the proof of Theorem 1.2 .

Proof of Theorem 1.2. Let $A \subset \mathcal{H}^{\sigma}\left(\mathbb{T}^{2}\right), \sigma \in(1, s)$, be a measurable set such that $\mu_{s}(A)=$ 0 . Then, for any $r>0$, we have

$$
\mu_{s, r}(A)=0
$$


By the mutual absolute continuity of $\mu_{s, r}$ and $\rho_{s, r}$, we obtain

$$
\rho_{s, r}(A)=0 \text {. }
$$

Then, by Lemma 5.5, we have

$$
\rho_{s, r}(\Phi(t)(A))=0
$$

for $t \in\left[0, t_{r}\right]$. By iterating this argument, we conclude that (5.10) holds for any $t>0$. By invoking the mutual absolute continuity of $\mu_{s, r}$ and $\rho_{s, r}$ once again, we have

$$
\mu_{s, r}(\Phi(t)(A))=0 .
$$

Finally, the dominated convergence theorem yields

$$
\mu_{s}(\Phi(t)(A))=\lim _{r \rightarrow \infty} \mu_{s, r}(\Phi(t)(A))=0 .
$$

By the time reversibility of the equation (1.8), the same conclusion holds for any $t<0$. This completes the proof of Theorem 1.2.

Remark 5.6. By combining Lemma [5.2 with the Yudovich's argument [30] as in [27, 18] (but with the critical power $p^{1}$ ), we can obtain the following quantitative bound, characterizing the quasi-invariance of $\rho_{s, r}$ :

$$
\rho_{s, r}(\Phi(t)(A)) \lesssim\left(\rho_{s, r}(A)\right)^{\frac{1}{c^{1+|t|}}}
$$

for any $t \in \mathbb{R}$. Here, the constant $c=c(r)$ depends on $r>0$.

\section{Quasi-InVARIANCE Under the NLW DYNAMiCS}

As already mentioned, the proof of Theorem 1.1 for the nonlinear wave equation is very close to that of Theorem 1.2 that we just presented in the previous section. In this section, we only explain the needed modifications.

6.1. The modified Gaussian measures. Since the quadratic part of the Hamiltonian $H$ defined in (1.3) for the nonlinear wave equation does not control the $L^{2}$-norm, we shall prove the quasi-invariance for a small modification of $\mu_{s}$ that is absolutely continuous with respect to $\mu_{s}$.

Define $\widetilde{\mu}_{s}$ as the induced probability measure under the map:

$$
\omega \in \Omega \longmapsto\left(u^{\omega}(x), v^{\omega}(x)\right)
$$

with

$$
u^{\omega}(x)=g_{0}+\sum_{n \in \mathbb{Z}^{2} \backslash\{0\}} \frac{g_{n}(\omega)}{\left(|n|^{2}+|n|^{2 s+2}\right)^{\frac{1}{2}}} e^{i n \cdot x} \quad \text { and } \quad v^{\omega}(x)=\sum_{n \in \mathbb{Z}^{2}} \frac{h_{n}(\omega)}{\left(1+|n|^{2 s}\right)^{\frac{1}{2}}} e^{i n \cdot x},
$$

where $\left\{g_{n}\right\}_{n \in \mathbb{Z}^{2}}$ and $\left\{h_{n}\right\}_{n \in \mathbb{Z}^{2}}$ are as in (1.5). With $\widehat{u}(0)=\int_{\mathbb{T}^{2}} u d x$, we can formally write $\widetilde{\mu}_{s}$ as

$$
d \widetilde{\mu}_{s}=Z_{s}^{-1} e^{-\frac{1}{2} \int v^{2}-\frac{1}{2} \int\left(D^{s} v\right)^{2}-\frac{1}{2}\left(\int u\right)^{2}-\frac{1}{2} \int|\nabla u|^{2}-\frac{1}{2} \int\left(D^{s+1} u\right)^{2}} d u d v,
$$

where

$$
D:=\sqrt{-\Delta}
$$

As we shall see below, the expression

$$
H_{0}(u, v)=\frac{1}{2} \int_{\mathbb{T}^{2}} v^{2}+\frac{1}{2} \int_{\mathbb{T}^{2}}\left(D^{s} v\right)^{2}+\frac{1}{2}\left(\int_{\mathbb{T}^{2}} u\right)^{2}+\frac{1}{2} \int_{\mathbb{T}^{2}}|\nabla u|^{2}+\frac{1}{2} \int_{\mathbb{T}^{2}}\left(D^{s+1} u\right)^{2}
$$


appears as the quadratic part of the renormalized energy in the context of the nonlinear wave equation. We have the following statement.

Lemma 6.1. Let $s>\frac{1}{2}$. Then, the Gaussian measures $\mu_{s}$ and $\widetilde{\mu}_{s}$ are equivalent.

Remark 6.2. In view of Lemma 6.1, it suffices to study the quasi-invariance property of $\widetilde{\mu}_{s}$ under the flow of the defocusing cubic nonlinear wave equation.

Proof. Note that $\mu_{s}$ and $\widetilde{\mu}_{s}$ are product measures on $u$ and $v$. Define (formally) $\mu_{s}^{1}$ and $\mu_{s}^{2}$ by

$$
d \mu_{s}^{1}=Z^{-1} e^{-\frac{1}{2} \int\left(J^{s+1} u\right)^{2}} d u \quad \text { and } \quad d \mu_{s}^{2}=Z^{-1} e^{-\frac{1}{2} \int\left(J^{s} v\right)^{2}} d v .
$$

Then, we have $\mu_{s}=\mu_{s}^{1} \otimes \mu_{s}^{2}$. Similarly, by defining $\widetilde{\mu}_{s}^{1}$ and $\widetilde{\mu}_{s}^{2}$ by

$$
\begin{aligned}
& d \widetilde{\mu}_{s}^{1}=Z^{-1} e^{-\frac{1}{2}\left(\int u\right)^{2}-\frac{1}{2} \int|\nabla u|^{2}-\frac{1}{2} \int\left(D^{s+1} u\right)^{2}} d u, \\
& d \widetilde{\mu}_{s}^{2}=Z^{-1} e^{-\frac{1}{2} \int v^{2}-\frac{1}{2} \int\left(D^{s} v\right)^{2}} d v
\end{aligned}
$$

we have $\widetilde{\mu}_{s}=\widetilde{\mu}_{s}^{1} \otimes \widetilde{\mu}_{s}^{2}$. Hence, it suffices to prove that $\mu_{s}^{j}$ and $\widetilde{\mu}_{s}^{j}$ are equivalent, $j=1,2$.

First, let us consider the $j=1$ case. Given $\sigma<s$, define $\lambda_{n}$ and $\widetilde{\lambda}_{n}$ by

$$
\lambda_{n}=\frac{1}{\langle n\rangle^{2 s+2-2 \sigma}} \quad \text { and } \quad \tilde{\lambda}_{n}= \begin{cases}1, & \text { if } n=0, \\ \frac{\langle n\rangle^{2 \sigma}}{|n|^{2}+|n|^{2 s+2}} & \text { if } n \neq 0 .\end{cases}
$$

Then, $\mu_{s}^{1}$ and $\widetilde{\mu}_{s}^{1}$ are the Gaussian measures on $H^{\sigma}\left(\mathbb{T}^{2}\right)$ with the covariance operators $Q$ and $\widetilde{Q}$ given by $\widetilde{9}$

$$
Q e_{n}=\lambda_{n} e_{n} \quad \text { and } \quad \widetilde{Q} e_{n}=\widetilde{\lambda}_{n} e_{n},
$$

respectively, where $e_{n}(x)=e^{i n \cdot x}$. Now, define $S_{n}$ by

$$
S_{n}=\frac{\left(\lambda_{n}-\widetilde{\lambda}_{n}\right)^{2}}{\left(\lambda_{n}+\widetilde{\lambda}_{n}\right)^{2}}
$$

Then, by Kakutani's theorem [10] (or Feldman-Hájek theorem [7, 9]), it follows that $\mu_{s}^{1}$ and $\widetilde{\mu}_{s}^{1}$ are equivalent if and only if

$$
\sum_{n \in \mathbb{Z}^{2}} S_{n}<\infty
$$

Otherwise, they are singular.

For $n \neq 0$, we have

$$
S_{n}=\frac{\left(|n|^{2}+|n|^{2 s+2}-\langle n\rangle^{2 s+2}\right)^{2}}{\left(|n|^{2}+|n|^{2 s+2}+\langle n\rangle^{2 s+2}\right)^{2}} \sim \frac{\left(|n|^{2}+|n|^{2 s+2}-\left(1+|n|^{2}\right)^{s+1}\right)^{2}}{\langle n\rangle^{4 s+4}} .
$$

By the mean value theorem applied to $f(x)=x^{s+1}$, we have

$$
\left.|| n\right|^{2 s+2}-\left(1+|n|^{2}\right)^{s+1}|=| f\left(|n|^{2}\right)-\left.f\left(1+|n|^{2}\right)|\sim| n\right|^{2 s} .
$$

\footnotetext{
${ }^{9}$ Namely, $Q$ and $\widetilde{Q}$ are defined by the following relations:

$-\frac{1}{2} \int\left(J^{s+1} u\right)^{2}=-\frac{1}{2}\left\langle Q^{-1} u, u\right\rangle_{H^{\sigma}} \quad$ and $\quad-\frac{1}{2}\left(\int u\right)^{2}-\frac{1}{2} \int\left(u^{2}+|\nabla u|^{2}+\left(D^{s+1} u\right)^{2}\right)=-\frac{1}{2}\left\langle\widetilde{Q}^{-1} u, u\right\rangle_{H^{\sigma}}$.
} 
Hence, we obtain

$$
S_{n} \sim \frac{\left(|n|^{2}+|n|^{2 s}\right)^{2}}{\langle n\rangle^{4 s+4}} \lesssim \frac{|n|^{4}+|n|^{4 s}}{\langle n\rangle^{4 s+4}}
$$

which is summable over $\mathbb{Z}^{2}$, provided that $s>\frac{1}{2}$. This proves (6.2) and the equivalence of $\mu_{s}^{1}$ and $\widetilde{\mu}_{s}^{1}$. A similar computation yields the equivalence of $\mu_{s}^{2}$ and $\widetilde{\mu}_{s}^{2}$. We omit details.

6.2. Renormalized energy for NLW. In this subsection, we derive the renormalized energy in the context of the truncated NLW:

$$
\left\{\begin{array}{l}
\partial_{t} u=v \\
\partial_{t} v=\Delta u-\pi_{N}\left(\left(\pi_{N} u\right)^{3}\right) .
\end{array}\right.
$$

Once the renormalized energy is derived, the remaining of the proof of Theorem 1.1 is exactly the same as the proof of Theorem 1.2

If $(u, v)$ is a solution to the truncated NLW (6.3), then we have

$$
\partial_{t}\left[\frac{1}{2} \int_{\mathbb{T}^{2}}\left(D^{s} v_{N}\right)^{2}+\frac{1}{2} \int_{\mathbb{T}^{2}}\left(D^{s+1} u_{N}\right)^{2}\right]=\int_{\mathbb{T}^{2}}\left(D^{2 s} v_{N}\right)\left(-u_{N}^{3}\right)
$$

where $\left(u_{N}, v_{N}\right)=\left(\pi_{N} u, \pi_{N} v\right)$ as before. Let $s \geq 2$ be an even integer. Then, by the Leibniz rule, we have

$$
\begin{aligned}
\int_{\mathbb{T}^{2}}\left(D^{2 s} v_{N}\right)\left(-u_{N}^{3}\right)= & -3 \int_{\mathbb{T}^{2}} D^{s} v_{N} D^{s} u_{N} u_{N}^{2} \\
& +\sum_{\substack{|\alpha|+|\beta|+|\gamma|=s \\
|\alpha|,|\beta|,|\gamma|<s}} c_{\alpha, \beta, \gamma} \int_{\mathbb{T}^{2}} D^{s} v_{N} \cdot \partial^{\alpha} u_{N} \cdot \partial^{\beta} u_{N} \cdot \partial^{\gamma} u_{N}
\end{aligned}
$$

for some inessential constants $c_{\alpha, \beta, \gamma}$. Furthermore, we can write

$$
\begin{aligned}
-3 \int_{\mathbb{T}^{2}} D^{s} v_{N} D^{s} u_{N} u_{N}^{2}=-\frac{3}{2} \partial_{t}\left[\int_{\mathbb{T}^{2}}\left(D^{s} u_{N}\right)^{2} u_{N}^{2}\right]+3 \int_{\mathbb{T}^{2}}\left(D^{s} u_{N}\right)^{2} v_{N} u_{N} \\
=-\frac{3}{2} \partial_{t}\left[\int_{\mathbb{T}^{2}} \mathbf{P}_{\neq 0}\left[\left(D^{s} u_{N}\right)^{2}\right] \mathbf{P}_{\neq 0}\left[u_{N}^{2}\right]\right]+3 \int_{\mathbb{T}^{2}} \mathbf{P}_{\neq 0}\left[\left(D^{s} u_{N}\right)^{2}\right] \mathbf{P}_{\neq 0}\left[v_{N} u_{N}\right] \\
-\frac{3}{2} \partial_{t}\left[\int_{\mathbb{T}^{2}}\left(D^{s} u_{N}\right)^{2} \int_{\mathbb{T}^{2}} u_{N}^{2}\right]+3 \int_{\mathbb{T}^{2}}\left(D^{s} u_{N}\right)^{2} \int v_{N} u_{N} .
\end{aligned}
$$

As in (1.26), the last two terms on the right-hand side are problematic. Therefore, we once again introduce a suitable renormalization. Define $\widetilde{\sigma}_{N}$ by

$$
\widetilde{\sigma}_{N}=\mathbb{E}_{\widetilde{\mu}_{s}}\left[\int_{\mathbb{T}^{2}}\left(D^{s} \pi_{N} u\right)^{2}\right]=\sum_{\substack{n \in \mathbb{Z}^{2} \\ 1 \leq|n| \leq N}} \frac{|n|^{2 s}}{|n|^{2}+|n|^{2 s+2}} \sim \log N .
$$

Then, we have

$$
\begin{aligned}
-\frac{3}{2} \partial_{t} & {\left[\int_{\mathbb{T}^{2}}\left(D^{s} u_{N}\right)^{2} \int u_{N}^{2}\right]+3 \int_{\mathbb{T}^{2}}\left(D^{s} u_{N}\right)^{2} \int v_{N} u_{N} } \\
& =-\frac{3}{2} \partial_{t}\left[\left(\int_{\mathbb{T}^{2}}\left(D^{s} u_{N}\right)^{2}-\widetilde{\sigma}_{N}\right) \int_{\mathbb{T}^{2}} u_{N}^{2}\right]+3\left(\int_{\mathbb{T}^{2}}\left(D^{s} u_{N}\right)^{2}-\widetilde{\sigma}_{N}\right) \int v_{N} u_{N} .
\end{aligned}
$$


Thanks to the Wiener chaos estimate (Lemma 3.2), the term

$$
\int_{\mathbb{T}^{2}}\left(D^{s} u_{N}\right)^{2}-\widetilde{\sigma}_{N}
$$

enjoys the bound

$$
\left\|\int_{\mathbb{T}^{2}}\left(D^{s} \pi_{N} u\right)^{2}-\widetilde{\sigma}_{N}\right\|_{L^{p}\left(d \widetilde{\mu}_{s}(u, v)\right)} \leq C p,
$$

for any finite $p \geq 2$, where the constant $C>0$ is independent of $p$ and $N$.

We now define the renormalized energy $H_{s, N}(u, v)$ by

$$
H_{s, N}(u, v)=\frac{1}{2} \int\left(D^{s} v\right)^{2}+\frac{1}{2} \int\left(D^{s+1} u\right)^{2}+\frac{3}{2} \int\left(D^{s} \pi_{N} u\right)^{2}\left(\pi_{N} u\right)^{2}-\frac{3}{2} \widetilde{\sigma}_{N} \int\left(\pi_{N} u\right)^{2} .
$$

Then, it follows from (6.4) - (6.7) that, if $(u, v)$ is a solution to (6.3), then we have

$$
\begin{aligned}
\partial_{t} H_{s, N}\left(u_{N}, v_{N}\right)= & 3 \int_{\mathbb{T}^{2}} \mathbf{P}_{\neq 0}\left[\left(D^{s} u_{N}\right)^{2}\right] \mathbf{P}_{\neq 0}\left[v_{N} u_{N}\right]+3\left(\int_{\mathbb{T}^{2}}\left(D^{s} u_{N}\right)^{2}-\widetilde{\sigma}_{N}\right) \int_{\mathbb{T}^{2}} v_{N} u_{N} \\
& +\sum_{\substack{|\alpha|+|\beta|+|\gamma|=s \\
|\alpha|,|\beta|,|\gamma|<s}} c_{\alpha, \beta, \gamma} \int_{\mathbb{T}^{2}} D^{s} v_{N} \cdot \partial^{\alpha} u_{N} \cdot \partial^{\beta} u_{N} \cdot \partial^{\gamma} u_{N} .
\end{aligned}
$$

As in Subsection 1.4, all terms on the right-hand-side of (6.8) are suitable for a perturbative analysis. However, a modification of the quadratic part is needed in order to have a resulting measure absolutely continuous with respect to $\widetilde{\mu}_{s}$.

For this purpose, we define the full renormalized energy $E_{s, N}(u, v)$ as

$$
E_{s, N}(u, v)=H_{s, N}(u, v)+H_{N}(u, v)+\frac{1}{2}\left(\int u d x\right)^{2},
$$

where $H_{N}$ is the conserved energy for the truncated NLW (6.3) defined by

$$
H_{N}(u, v):=\frac{1}{2} \int_{\mathbb{T}^{2}}\left(|\nabla u|^{2}+v^{2}\right) d x+\frac{1}{4} \int_{\mathbb{T}^{2}}\left(\pi_{N} u\right)^{4} d x .
$$

The quadratic part of $E_{s, N}$ is now given by (6.1), resulting in the Gaussian measure $\widetilde{\mu}_{s}$ equivalent to $\mu_{s}$. Using the truncated NLW (6.3), we have that

$$
\partial_{t} E_{s, N}\left(u_{N}, v_{N}\right)=\partial_{t} H_{s, N}\left(u_{N}, v_{N}\right)+\left(\int_{\mathbb{T}^{2}} u_{N}\right)\left(\int_{\mathbb{T}^{2}} v_{N}\right) .
$$

Hence, the only new term to be handled as compared to the proof of Theorem 1.2 is

$$
\left(\int_{\mathbb{T}^{2}} u_{N}\right)\left(\int_{\mathbb{T}^{2}} v_{N}\right)
$$

More precisely, we need to estimate (6.10) under the restriction on the truncated energy

$$
H_{N}(u, v) \leq r .
$$

By the compactness of the domain $\mathbb{T}^{2}$, we have

$$
\left|\left(\int_{\mathbb{T}^{2}} u_{N}\right)\left(\int_{\mathbb{T}^{2}} v_{N}\right)\right| \leq\left\|\pi_{N} u\right\|_{L^{4}\left(\mathbb{T}^{2}\right)}\left\|\pi_{N} v\right\|_{L^{2}\left(\mathbb{T}^{2}\right)} \leq C_{r}
$$

under (6.11). Therefore, the contribution of (6.10) to $\partial_{t} E_{s, N}\left(u_{N}, v_{N}\right)$ is easy to deal with. We finally note that the introduction of $H_{N}(u, v)$ in the definition (6.9) of the modified energy leads to the introduction of a new harmless term $\int\left(\pi_{N} u\right)^{4}$ in the definition of the 
weighted Gaussian measures $\rho_{s, N, r}$. The remaining part of the analysis leading to the proof of Theorem 1.1 is exactly the same 10 as the one already presented in the proof of Theorem 1.2 and therefore we omit details.

\section{Appendix A. On the Dispersion generalized NLKG}

In this appendix, we briefly discuss the situation for the (much easier) dispersion generalized NLKG (1.33) with $\beta>1$. The equation (1.33) is a Hamiltonian equation with the Hamiltonian given by

$$
E^{\beta}(u)=\frac{1}{2} \int_{\mathbb{T}^{2}}\left(J^{\beta} u\right)^{2}+\frac{1}{2} \int_{\mathbb{T}^{2}} v^{2}+\frac{1}{4} \int_{\mathbb{T}^{2}} u^{4} .
$$

By repeating the computation in Subsection 1.4, we have

$$
\begin{aligned}
\partial_{t}\left[\frac{1}{2} \int_{\mathbb{T}^{2}}\left(J^{s} v\right)^{2}\right. & \left.+\frac{1}{2} \int_{\mathbb{T}^{2}}\left(J^{s+\beta} u\right)^{2}\right]=\int_{\mathbb{T}^{2}} J^{2 s} v\left(-u^{3}\right) \\
& =-3 \int_{\mathbb{T}^{2}}\left(\partial_{t} J^{s} u\right) J^{s} u \cdot u^{2}+\text { l.o.t. } \\
& =-\frac{3}{2} \partial_{t}\left[\int_{\mathbb{T}^{2}}\left(J^{s} u\right)^{2} u^{2}\right]+3 \int_{\mathbb{T}^{2}}\left(J^{s} u\right)^{2} \partial_{t} u \cdot u+\text { l.o.t. }
\end{aligned}
$$

where "l.o.t." denotes various (insignificant) lower order terms. Define $E_{s}^{\beta}(u, v)$ and $E_{s, N}^{\beta}(u, v)$ by

$$
\begin{aligned}
E_{s}^{\beta}(u, v) & =\frac{1}{2} \int\left(J^{s} \partial_{t} u\right)^{2}+\frac{1}{2} \int\left(J^{s+\beta} u\right)^{2}+\frac{3}{2} \int\left(J^{s} u\right)^{2} u^{2}, \\
E_{s, N}^{\beta}(u, v) & =\frac{1}{2} \int\left(J^{s} \partial_{t} u\right)^{2}+\frac{1}{2} \int\left(J^{s+\beta} u\right)^{2}+\frac{3}{2} \int\left(J^{s} \pi_{N} u\right)^{2}\left(\pi_{N} u\right)^{2} .
\end{aligned}
$$

Define the following weighted Gaussian measure $\rho_{s, N, r}^{\beta}, N \in \mathbb{N} \cup\{\infty\}$, by

$$
d \rho_{s, N, r}^{\beta}=Z^{-1} \mathbf{1}_{\left\{E_{N}^{\beta}(u, v) \leq r\right\}} e^{-E_{s, N}^{\beta}(u, v)} d u d v=Z^{-1} \mathbf{1}_{\left\{E_{N}^{\beta}(u, v) \leq r\right\}} e^{-\frac{3}{2} \int\left(J^{s} \pi_{N} u\right)^{2}\left(\pi_{N} u\right)^{2}} d \mu_{s}^{\beta},
$$

where $\mu_{s}^{\beta}$ is as in (1.34) and $E_{N}^{\beta}$ is the truncated energy defined by

$$
E_{N}^{\beta}(u)=\frac{1}{2} \int_{\mathbb{T}^{2}}\left(J^{\beta} u\right)^{2}+\frac{1}{2} \int_{\mathbb{T}^{2}} v^{2}+\frac{1}{4} \int_{\mathbb{T}^{2}}\left(\pi_{N} u\right)^{4} .
$$

Then, in view of the comment in Remark 1.8, we can repeat the argument in Section 3 (without any renormalization) and show that $\rho_{s, N, r}^{\beta}$ is a well defined probability measure (even when $r=\infty$ thanks to the defocusing nature of the equation) with a uniform bound in $N \in \mathbb{N} \cup\{\infty\}$.

\footnotetext{
${ }^{10}$ Note that the proof of the change-of-variable formula (an analogue of Lemma 5.1 for NLW) requires (i) the Hamiltonian structure of the truncated dynamics (6.3), leading to the invariance of the Lebesgue measure $L_{N}$ on $\mathcal{E}_{N} \times \mathcal{E}_{N}$ and (ii) invariance of the marginal Gaussian measure $\widetilde{\mu}_{s, N}^{\perp}$ on $\pi_{N}^{\perp} \mathcal{H}^{\sigma}\left(\mathbb{T}^{2}\right)$. See the proofs of Proposition 4.1 in [27] and Proposition 6.6 in [18. Clearly, (i) is satisfied. We see that (ii) is also satisfied since $H_{0}$ defined in (6.1) satisfies$$
H_{0}\left(\pi_{N}^{\perp} u, \pi_{N}^{\perp} v\right)=\frac{1}{2} \int_{\mathbb{T}^{2}}\left(\pi_{N}^{\perp} v\right)^{2}+\frac{1}{2} \int_{\mathbb{T}^{2}}\left(D^{s} \pi_{N}^{\perp} v\right)^{2}+\frac{1}{2} \int_{\mathbb{T}^{2}}\left|\nabla \pi_{N}^{\perp} u\right|^{2}+\frac{1}{2} \int_{\mathbb{T}^{2}}\left(D^{s+1} \pi_{N}^{\perp} u\right)^{2}
$$

which is conserved by the linear wave dynamics on the high frequencies $\left(\mathcal{E}_{N} \times \mathcal{E}_{N}\right)^{\perp}$.
} 
Let us now turn to the energy estimate. Let $s \geq \beta>1$. It follows from (A.1), (A.2), and (A.3) that

$$
\partial_{t} E_{s, N}^{\beta}(u, v)=3 \int\left(J^{s} \pi_{N} u\right)^{2} \cdot \pi_{N} v \cdot \pi_{N} u+\text { l.o.t. }
$$

for a solution $(u, v)$ to the following truncated dispersion generalized NLKG:

$$
\left\{\begin{array}{l}
\partial_{t} u=v \\
\partial_{t} v=J^{2 \beta} u-\pi_{N}\left(\left(\pi_{N} u\right)^{3}\right) .
\end{array}\right.
$$

By interpolation and the Sobolev embedding $H^{\beta}\left(\mathbb{T}^{2}\right) \subset L^{\infty}\left(\mathbb{T}^{2}\right), \beta>1$, we have

$$
\begin{aligned}
\left|\int_{\mathbb{T}^{2}}\left(J^{s} \pi_{N} u\right)^{2} \cdot \pi_{N} v \cdot \pi_{N} u\right| & \leq\left\|J^{s} \pi_{N} u\right\|_{L_{x}^{4}}^{2} \underbrace{\left\|\pi_{N} v\right\|_{L_{x}^{2}}\left\|\pi_{N} u\right\|_{L_{x}^{\infty}}}_{\underbrace{\beta}_{N}(u, v)} \\
& \lesssim\left\|J^{s+\beta-1-\varepsilon} \pi_{N} u\right\|_{L_{x}^{r}}^{2(1-\theta)}\left(E_{N}^{\beta}(u, v)\right)^{1+2 \theta},
\end{aligned}
$$

for some $\theta \in(0,1]$ and $r>4$ satisfying

$$
s=\theta \beta+(1-\theta)(s+\beta-1-\varepsilon) \quad \text { and } \quad \frac{1}{4}=\frac{\theta}{2}+\frac{1-\theta}{r} .
$$

Hence, by the Wiener chaos estimate (Lemma 3.2 ), we obtain the crucial energy estimate:

$$
\left\|\mathbf{1}_{\left\{E_{N}^{\beta}(u, v) \leq r\right\}} \cdot \int_{\mathbb{T}^{2}}\left(J^{s} \pi_{N} u\right)^{2} \cdot \pi_{N} v \cdot \pi_{N} u\right\|_{L^{p}\left(d \mu_{s}^{\beta}\right)} \lesssim p^{1-\theta}
$$

for some $\theta>0$. The lower order terms in (A.4) can be handled in a similar (or easier) manner. Then, one can repeat the argument in [27] and prove quasi-invariance of the Gaussian measure $\mu_{s}^{\beta}$, at least for an even integer $s \geq \beta$.

Acknowledgements. T.O. was supported by the European Research Council (grant no. 637995 "ProbDynDispEq"). The authors would like to thank Prof. Andrew Stuart for pointing out the remark on propagation of additional regularity in Subsection 1.3. Prof. David Elworthy for pointing out the references [20, 21], and Prof. Sergey Nazarenko for an interesting discussion on wave turbulence. The authors are also grateful to the anonymous referees for their helpful comments that have improved the presentation of this paper.

\section{REFERENCES}

[1] J. Bourgain, Invariant measures for the 2D-defocusing nonlinear Schrödinger equation, Comm. Math. Phys. 176 (1996), no. 2, 421-445.

[2] J. Bourgain, Gibbs measures and quasi-periodic solutions for nonlinear Hamiltonian partial differential equations, The Gelfand Mathematical Seminars, 1993-1995, 23-43, Gelfand Math. Sem., Birkhäuser Boston, Boston, MA, 1996.

[3] R. Cameron, W. Martin, Transformations of Wiener integrals under translations, Ann. of Math. 45, (1944). 386-396.

[4] A.B. Cruzeiro, Équations différentielles ordinaires: non explosion et mesures quasi-invariantes, (French) J. Funct. Anal. 54 (1983), no. 2, 193-205.

[5] A.B. Cruzeiro, Équations différentielles sur l'espace de Wiener et formules de Cameron-Martin nonlinéaires, (French) J. Funct. Anal. 54 (1983), no. 2, 206-227.

[6] G. Da Prato, L. Tubaro, Wick powers in stochastic PDEs: an introduction, Technical Report UTM, 2006, 39 pp. 
[7] J. Feldman Equivalence and perpendicularity of Gaussian processes, Pacific J. Math. 8 (1958) 699-708.

[8] L. Gross, Abstract Wiener spaces, Proc. 5th Berkeley Sym. Math. Stat. Prob. 2 (1965), 31-42.

[9] J. Hájek On a property of normal distribution of any stochastic process, (Russian. English summary) Czechoslovak Math. J. 8 (83) (1958) 610-618.

[10] S. Kakutani, On equivalence of infinite product measures, Ann. of Math. 49 (1948), 214-224.

[11] H. Kuo, Gaussian measures in Banach spaces, Lecture Notes in Mathematics, Vol. 463. Springer-Verlag, Berlin-New York, 1975. vi+224 pp.

[12] S. Nazarenko, Wave turbulence, Lecture Notes in Physics, 825. Springer, Heidelberg, 2011. xvi+279 pp.

[13] E. Nelson, A quartic interaction in two dimensions, 1966 Mathematical Theory of Elementary Particles (Proc. Conf., Dedham, Mass., 1965) pp. 69-73 M.I.T. Press, Cambridge, Mass.

[14] T. Oh, P. Sosoe, N. Tzvetkov, An optimal regularity result on the quasi-invariant Gaussian measures for the cubic fourth order nonlinear Schrödinger equation, J. Éc. polytech. Math. 5 (2018), 793-841.

[15] T. Oh, L. Thomann, A pedestrian approach to the invariant Gibbs measures for the 2-d defocusing nonlinear Schrödinger equations, Stoch. Partial Differ. Equ. Anal. Comput. 6 (2018) 397-445.

[16] T. Oh, L. Thomann, Invariant Gibbs measures for the 2-d defocusing nonlinear wave equations, to appear in Ann. Fac. Sci. Toulouse Math.

[17] T. Oh, Y. Tsutsumi, N. Tzvetkov, Quasi-invariant Gaussian measures for the cubic nonlinear Schrödinger equation with third order dispersion, arXiv:1805.08409 [math.AP].

[18] T.Oh, N. Tzvetkov, Quasi-invariant Gaussian measures for the cubic fourth order nonlinear Schrödinger equation, Probab. Theory Relat. Fields 169 (2017), 1121-1168.

[19] T. Oh, N. Tzvetkov, On the transport of Gaussian measures under the flow of Hamiltonian PDEs, Séminaire Laurent Schwartz-Équations aux dérivées partielles et applications. Année 2015-2016, Exp. No. VI, 9 pp., Ed. Éc. Polytech., Palaiseau, 2017.

[20] G. Peters, Flows on the Wiener space generated by vector fields with low regularity, C. R. Acad. Sci. Paris Sér. I Math. 320 (1995), no. 8, 1003-1008.

[21] G. Peters, Symplectic geometry on an abstract Wiener space and the sine-Gordon equation, C. R. Acad. Sci. Paris Sér. I Math. 320 (1995), no. 9, 1123-1128.

[22] R. Ramer, On nonlinear transformations of Gaussian measures, J. Functional Analysis 15 (1974), 166187.

[23] B. Simon, The $P(\varphi)_{2}$ Euclidean (quantum) field theory, Princeton Series in Physics. Princeton University Press, Princeton, N.J., 1974. xx+392 pp.

[24] L. Thomann, N. Tzvetkov, Gibbs measure for the periodic derivative nonlinear Schrödinger equation, Nonlinearity 23 (2010), no. 11, 2771-2791.

[25] N. Tzvetkov, Construction of a Gibbs measure associated to the periodic Benjamin-Ono equation, Probab. Theory Related Fields 146 (2010), no. 3-4, 481-514.

[26] N. Tzvetkov, Invariant measures for the defocusing Nonlinear Schrödinger equation (Mesures invariantes pour l'équation de Schrödinger non linéaire), Annales de l'Institut Fourier, 58 (2008), 2543-2604.

[27] N. Tzvetkov, Quasi-invariant Gaussian measures for one dimensional Hamiltonian PDE's, Forum Math. Sigma 3 (2015), e28, 35 pp.

[28] N. Tzvetkov, N. Visciglia, Gaussian measures associated to the higher order conservation laws of the Benjamin-Ono equation, Ann. Sci. Éc. Norm. Supér. 46 (2013), no. 2, 249-299.

[29] N. Tzvetkov, N. Visciglia, Invariant measures and long-time behavior for the Benjamin-Ono equation, Int. Math. Res. Not. IMRN (2014), no. 17, 4679-4714.

[30] V. Yudovich, Non-stationary flows of an ideal incompressible fluid, Zh. Vychisl. Math. i Math. Fiz. (1963) 1032-1066 (in Russian).

[31] V. Zakharov, V. L'vov, G. Falkovich, Kolmogorov Spectra of Turbulence I, Wave Turbulence, Springer Series in Nonlinear Dynamics, Springer-Verlag, Berlin Heidelberg, 1992. xiii+264 pp.

Tadahiro Oh, School of Mathematics, The University of Edinburgh, and The Maxwell Institute for the Mathematical Sciences, James Clerk Maxwell Building, The King's Buildings, Peter Guthrie Tait Road, Edinburgh, EH9 3FD, United Kingdom

E-mail address: hiro.oh@ed.ac.uk

Nikolay Tzvetkov, Université de Cergy-Pontoise, 2, av. Adolphe Chauvin, 95302 CergyPontoise Cedex, France

E-mail address: nikolay.tzvetkov@u-cergy.fr 